\title{
Melatonin Alleviates Microglia-Mediated Neuroinflammation By Suppressing NLRP3 Inflammasome-Mediated Pyroptosis Via ROS/mtDNA/STING Pathway After Spinal Cord Injury
}

Jin Wang

Huashan Hospital Fudan University

Haiyuan Yang

Huashan Hospital Fudan University

Fan Zhang

Huashan Hospital Fudan University

Minghao Shao

Huashan Hospital Fudan University

Haocheng Xu

Huashan Hospital Fudan University

Shun Xu

Huashan Hospital Fudan University

FeiZhou Lyu ( $D$ lyufzsubmit@163.com )

Huashan Hospital Fudan University

\section{Research}

Keywords: spinal cord injury, melatonin, neuroinflammation, NLRP3 inflammasome, pyroptosis, ROS/mtDNA/STING pathway

Posted Date: July 26th, 2021

DOI: https://doi.org/10.21203/rs.3.rs-716835/v1

License: (9) This work is licensed under a Creative Commons Attribution 4.0 International License.

Read Full License 


\section{Abstract}

\section{Background}

Microglia pyroptosis-induced neuroinflammation has been one of the potential treatment targets for spinal cord injury (SCl). And melatonin is reported to have anti-neuroinflammation effect on $\mathrm{SCl}$, but the underlying mechanism is largely unexplored. In addition, the potential regulatory role of stimulator of interferon genes (STING) mediated innate immune response in the SCl-induced neuroinflammation also remains unknown. The aim of this study is to identify the potential molecular mechanism of the antineuroinflammation effect of melatonin in SCI mice and to explore whether STING-mediated signal pathway is involved in this pharmacological process.

\section{Methods}

In vivo, the C57BL/6 female mice underwent SCl injury or Sham surgery (laminectomy alone). Melatonin and selective STING antagonist C-176 were administered intraperitoneally after injury in the SCl group once a day for 3 or 28 consecutive days for different experiments. The BMS score system was adopted to assess the motor function of mice. In vitro, the Lipopolysaccharide (LPS)/ATP was combinedly used to induce cell pyroptosis in BV2 microglia and the adenovirus was used to overexpress STING. A series of molecular experiments including Western blot (WB), quantitative real-time polymerase chain reaction (RTqPCR), enzyme linked immunosorbent assay (ELISA) and immunofluorescence (IF) were performed in vivo and in vitro.

\section{Results}

Our results showed that melatonin effectively suppressed NLRP3 inflammasome-induced pyroptosis and STING-mediated pathway after SCI. In addition, C-176 also alleviated the NLRP3 inflammasomemediated pyroptosis and promoted functional recovery in vivo. In vitro, we also found that melatonin abrogated NLRP3 inflammasome activation in LPS/ATP-induced BV2 cells, while overexpression of STING reversed the anti-pyroptotic role of melatonin. Subsequent results together indicated that the role of melatonin on STING-dependent NLRP3 inflammasome activation may be mediated by decreasing ROS production and cytosolic mtDNA release.

\section{Conclusion}

This study preliminarily demonstrated that melatonin exerts its anti-neuroinflammation role on $\mathrm{SCl}$ by alleviating the NLRP3 inflammasome-mediated pyroptosis, which was mediated by blocking the ROS/mtDNA/STING pathway. It provides us a better understanding of the pathological mechanism after $\mathrm{SCl}$ and offer experiment evidence to promote the use of melatonin for $\mathrm{SCl}$.

\section{Introduction}


Spinal cord injury $(\mathrm{SCl})$ is one of the most common and fatal injury of the spine, which leads to limb paralysis and other severe complications including sensory disorders, bedsore and lung infection[1]. There are approximately 2.5 million SCI patients around the world and an increase of 130,000 annually[2]. Unfortunately, there is no ideal or effective treatments for $\mathrm{SCl}$ in clinic for its complex pathological mechanism $[3,4]$. Nowadays, it is widely accepted that the neuroinflammation-mediated extensive neuronal damage during the secondary phase of $\mathrm{SCl}$ is the main cause of persistent destruction after injury $[5,6]$. Therefore, how to attenuate the neuroinflammation has become a hot issue for the research of SCl.

The NOD-like Receptor protein 3 (NLRP3) inflammasome, a cytosolic molecular platform comprised by NLRP3, apoptosis associated speck-like protein containing a caspase recruitment domain (ASC) and procaspase-1, can be activated by both the pathogen-associated molecular patterns (PAMPs) and damageassociated molecular patterns (DAMPs) [7, 8]. Once NLRP3 inflammasome activated, the caspase-1dependent Gasdermin D (GSDMD) cleavage leads to the pore formation on the cell membrane and subsequently promotes the secretion of mature proinflammatory cytokines (IL-1 $\beta$ and IL-18), which will greatly exaggerate the inflammation response. This kind of programmed cell death is also known as cell pyroptosis[9]. Recent researches have shown that NLRP3-mediated pyroptosis plays crucial roles in various diseases including intestinal diseases[10], cardiovascular diseases[11, 12], neurological disease[13] as well as the age-related diseases[14]. Importantly, some researchers and we previously reported that NLRP3 inflammasome-mediated pyroptosis is also involved in $\mathrm{SCl}$ and the inhibition of pyroptosis may be one of the potential treatment targets for SCI[15-17].

Cyclic GMP-AMP synthase (cGAS)-stimulator of interferon genes (STING) axis, a newly identified signaling pathway, functions as an innate immune defensive system by sensing cytosolic DNA[18, 19]. In brief, DNA in the cytosol activates cGAS and produces cGAMP (cyclic GMP-AMP), which functions as a secondary messenger that binds and activates the STING. Then the downstream kinases such as tankbinding kinase 1 (TBK1) will be recruited to phosphorylate and facilitate nuclear translocation of the transcription factors (IRF3 and NF-kB) that play corresponding roles[20, 21]. Recently, increasing evidences pointed that STING participates in multiple diseases by regulating NLRP3 inflammasome[22, 23]. Li N[24] unveiled that STING could activate the NLRP3 inflammasome and pyroptosis in cardiomyocytes. Notably, they also reported that STING was involved in the development of LPS-induced acute lung injury by promoting the activation NLRP3 inflammasome and pyroptosis[25]. However, whether STING can regulate NLRP3 inflammasome-mediated pyroptosis in SCI has not been well investigated till now.

Melatonin ((N-acetyl-5-methoxytryptamine), a neuroendocrine hormone which was primarily synthesized in the pineal gland, has been frequently reported to have protective effects on various diseases for its diversity functions such as antioxidant, circadian

rhythm regulation, sleep promotion and immunomodulatory effects[26-31]. Of note, melatonin has been reported to own great anti-inflammatory function by supressing NLRP3 inflammasome-mediated 
pyroptosis[32-34]. Liu Z [35] reported that melatonin could prevent and treat systemic inflammatory response via alleviating NLRP3 inflammasome-induced pyroptosis by blocking NF-KB/GSDMD signal in mice adipose tissue. Zhang $Y$ [36] also pointed that melatonin replacement strategy might be considered as a new method to treat atherosclerosis for its essential role in against endothelial pyroptosis. However, fewer evidences were reported about the regulatory role of melatonin on NLRP3 inflammasome-mediated pyroptosis after $\mathrm{SCl}$. In most recently, melatonin is reported to delay aging and neurodegeneration by inhibiting the cytosolic mtDNA-activated cGAS/STING/IRF3 pathway[37]. It greatly encouraged us to speculate that if mIDNA-activated STING pathway is also involved in the process of melatonin on regulating pyroptosis in $\mathrm{SCl}$.

Therefore, the aims of this study were to determine the role of melatonin on NLRP3 inflammasomemediated pyroptosis after SCI and to investigate whether the STING-mediated signaling pathway was involved in the underlying mechanism.

\section{Materials And Methods}

\subsection{Animals}

The C57BL/6 clean-grade female mice were purchased from the Animal center of Chinese Academy of Science (Shanghai, China), and housed in a humidity and temperature-controlled environment with free access to food and water $\left(20-25^{\circ} \mathrm{C}, 65 \%\right.$ humidity, $12 \mathrm{~h}$ light/dark cycle). All animal experiments involved in this study followed the guidelines established by the Animal Ethical and Welfare Committee of Huashan Hospital, Fudan University.

\subsection{Mouse model of SCl}

The SCl mice model was prepared following the protocol as described in our previous research[5]. In brief, C57BL/ 6 female mice were weighed and anesthetized with intraperitoneal injection of $4 \%$ pentobarbital sodium $(35 \mathrm{mg} / \mathrm{kg})$. The T8-T9 vertebrae were removed by laminectomy and the spinal cord was exposed. The mice in SCl group were inflicted by laterally compressing the cord using Dumont-type forceps with a spacer of $0.2 \mathrm{~mm}$ for $20 \mathrm{~s}$. The mice in Sham group received laminectomy alone without injury to the spinal cord. All the wounds were washed with sterile saline and sutured layer by layer. Following surgery, Penicillin $(20,000 \mathrm{U})$ was injected intramuscularly once a day for 3 consecutive days and bladder massage was conducted manually 3 times a day to assist urination.

After operation, the mice in SCl-Melatonin group received daily intraperitoneal injections of $10 \mathrm{mg} / \mathrm{kg}$ melatonin (Sigma-Aldrich, St. Louis, MO, USA) for 3 days (for histological analysis) or 28 days (for BMS score analysis). In SCI-C176 group, the STING antagonist C-176 (MedChemExpress, 750nmol dissolved in $200 \mu \mathrm{L}$ corn oil) was firstly injected intraperitoneally at $1 \mathrm{~h}$ post-injury [38], and then be used as the same way as melatonin.

\subsection{Assessment for motor function of mice}


The Basso Mouse Scale (BMS) score (0 to 9 points) was recorded at 1, 3, 5, 7, 14, 21 and 28 days after surgery to assess the locomotion function of the mice[39]. Briefly, the mice were allowed to move freely for 5 minutes in an open field, and the hindlimb movements, stepping and coordination points were assessed by two investigators who were blinded to treatment.

\subsection{Cell culture and drug treatments}

The murine BV2 microglia cell line was purchased from the Cell Bank of the Chinese Academy of Science (Shanghai, China) and cultured in DMEM medium (Gibco, Carlsbad, CA, USA) containing $10 \%$ fetal bovine serum (FBS; Gibco, Carlsbad, CA, USA) and an antibiotic mixture (1\% penicillin/streptomycin) (Invitrogen, Carlsbad, CA, USA) at $37^{\circ} \mathrm{C}$ and $5 \% \mathrm{CO}_{2}$.

The cells were randomly assigned into the control and the LPS/ATP treatment groups. In LPS/ATP group, BV2 cells were exposed to the $1000 \mathrm{ng} / \mathrm{ml}$ LPS (Sigma-Aldrich, St. Louis, MO, USA) for $4 \mathrm{~h}$, followed by $5 \mathrm{mM}$ ATP (Sigma-Aldrich, St. Louis, MO, USA) for $2 \mathrm{~h}$ to induce pyroptosis. In Melatonin group, the cells were pre-treated with melatonin at the concentration of $5 \mu \mathrm{M}$ or $10 \mu \mathrm{M}$ for $24 \mathrm{~h}$ before LPS/ATP stimulation.

The adenoviral infection was adopted to overexpress STING in BV2 cells according to the manufacturers' instructions. Adv-control or Adv-STING were designed and synthesized by Han Heng (Shanghai, China). Briefly, when cells reached $60 \%$ confluence, they were infected with Adv-control or Adv-STING at different multiplicity of infection (MOI), followed by incubation for $6 \mathrm{~h}$. Then, the culture medium was replaced with fresh completed DMEM.

\subsection{Cell viability assay}

The cell viability of BV2 cells after melatonin treatment was assessed by CCK-8 assay (Sigma-Aldrich, MO, USA) according to the manufacture's protocol. Briefly, BV2 cells were seeded in the 96-well plates and cultured for $24 \mathrm{~h}$, followed by a series concentration of melatonin $(5,10,100,500,1000 \mu \mathrm{M})$ treatment for $24 \mathrm{~h}$. Then the culture medium was removed and $110 \mu \mathrm{L} \mathrm{CCK} 8$ work solution (containing $10 \mu \mathrm{L}$ CCK8 reagent) was added in each well. After incubation for $2 h$, the absorbance of each well was detected at $450 \mathrm{~nm}$.

\subsection{Cell Morphological observation}

Following drug treatment (LPS/ATP, melatonin), the medium was removed and the $4 \%$ formaldehyde was added for $30 \mathrm{~min}$. After that, cells washed with PBS for 3 times (10min per time), then the morphology of BV2 cells was photographed under light microscope.

\subsection{Propidium iodide staining}

After corresponding treatment, BV2 cells were stained with propidium iodide staining $(\mathrm{PI})$ and Hoechst for 15min and the PI positive cells were observed under the fluorescence microscope (Leica, Germany). 


\subsection{Cell death assay}

The secreted levels of lactate dehydrogenase (LDH) were detected using the LDH Cytotoxicity Assay Kit (Beyotime, Shanghai, China) according to the manufacturer's instructions.

\subsection{Enzyme-linked immunosorbent assay (ELISA)}

The levels of IL-1 $\beta$, IL-18 and TNF- $\alpha$ in cell culture supernatants and mouse serum were measured using commercial ELISA kits (Sigma-Aldrich, St. Louis, MO, USA) according to the manufacturer's protocol. The absorbance was detected by microplate reader (Thermo Scientific, Waltham, MA, USA) at $450 \mathrm{~nm}$ wavelength.

\subsection{Measurement of cGAMP}

cGAMP in spinal cord tissue and cell lysate was measured by chemiluminescent enzyme immunoassay (2'3'-cGAMP ELISA kit, Cayman Chemical, USA) according to manufacturers' protocols.

\subsection{RNA extraction and quantitative real-time PCR}

Total RNA was extracted from the spinal cord tissue or the harvested BV2 cells using Trizol reagent (Invitrogen, San Diego, CA, USA) and reverse-transcribed, then cDNAs were amplified according to the manufacturer's instruction. The SYBR Green PCR Master Mix Kit (Applied Biosystems, Shanghai, CHINA) was used to quantify the relative mRNA levels. $\beta$-actin served as the internal controls and the $2^{-\triangle \Delta C T}$ method was adopted to analyze the relative expression levels of mRNAs. Primer sequences are listed in Table.1.

\subsection{Western blotting (WB) analysis}

Total protein was extracted from the spinal cord tissues and BV2 cells using RIPA buffer containing phosphatase and protease inhibitor cocktail, and then quantified by the BCA Protein Assay kit. Protein samples were separated by $10 \%$ SDS-PAGE gels and transferred to polyvinylidene difluoride membranes (Millipore, Billerica, MA, USA). After blocked with 5\% non-fat milk at room temperature for $1 \mathrm{~h}$, the membranes were incubated overnight at $4^{\circ} \mathrm{C}$ with primary antibodies: NLRP3 (1:1000, CST, 15101s), IL$1 \beta$ (1:1000, ABclonal, A16288), CASP-1 (1:1000, ABclonal, A18646), p65 (1:1000, CST, 8242), P-p65 (1:1000, CST, 3033), GSDMD (1:1000, Abcam, ab209845), ASC (1:1000, ABclonal, A11433), STING (1:1000, CST, 13647), TBK1 (1:2000, abcam, ab40676), P-TBK1 (1:1000, CST, 5483), IRF3 (1:1000, abcam, ab68481), P-IRF3 (1:1000, CST, 4947) and $\beta$-actin (1:2000, Abcam, ab8245). Following washed with TBST for 3 times, the HRP-conjugated secondary antibody (1:2000) was added and incubated at room temperature for $1 \mathrm{~h}$. The protein bands were visualized using an enhanced chemiluminescence (ECL) kit, and blots were quantified by the gel imaging system (UVP LLC, Upland, CA, USA).

\subsection{Histological analysis and immunofluorescence}


On the third day after $\mathrm{SCl}$, the mice $(\mathrm{n}=10)$ were sacrificed and the spinal cord segments near the lesion epicenter were harvested. The samples were then fixed with $4 \%$ paraformaldehyde overnight followed by embedded in paraffin. Hematoxylin and eosin (H\&E) staining was performed according to the manufacturer's instructions. For Nissl staining, the sections were stained with toluidine blue following a standard protocol.

Immunofluorescence analysis was performed as previously described[5]. The sections of spinal cord tissue or BV2 cells in each group were fixed with $4 \%$ paraformaldehyde for $30 \mathrm{~min}$ and then blocked in $5 \%$ bovine serum albumin (BSA) containing $0.3 \%$ Triton $X-100$. Then the samples were incubated with primary antibodies overnight at $4^{\circ} \mathrm{C}$ : GSDMD (1:100, Abcam, ab209845), NLRP3 (1:100, ABclonal, A12694), STING (1:100, Abcam, ab210070), IRF-3(1:100, ABclonal, A0816), A. On the second day, the samples were incubated with appropriate fluorescently labeled secondary antibodies (1:250, Molecular Probes) and counterstained with 4,6-diamidino-2-phenylindole dihydrochloride (DAPI, Sigma-Aldrich). All images were observed with an optical or fluorescence microscope (Leica, Germany).

\subsection{Measurement of ROS production}

Total ROS production was detected by commercial fluorescence probe. Briefly, BV2 cells pretreated with or without melatonin were stimulated by LPS/ATP and then incubated in the dark with $10 \mu \mathrm{M}$ DCFH-DA \MedChemExpress, USA \at $37^{\circ} \mathrm{C}$ for 30 min. Then cells were washed with PBS to remove the extracellular DCFH-DA followed by observing under the fluorescence microscope (Leica, Germany). Relative ROS production was quantified by the change of fluorescence intensity comparing to the control cells at isosmolar condition.

\subsection{5 mtDNA DNA isolation and mtDNA copy number analysis}

mtDNA was evaluated by RT-qPCR as previously described[40]. In brief, BV2 cells were harvested after corresponding treatment in different groups. Thereafter, cytosolic fractions were isolated by centrifugation, and the DNA was isolated from the whole cell extracts and cytosolic fractions respectively (Qiaquick nucleotide removal columns, Qiagen). The whole cell extracts served as control for total mtDNA.

\subsection{Statistical analysis}

All results are presented as means \pm standard deviation. Student's unpaired $t$ tests and one-way ANOVA

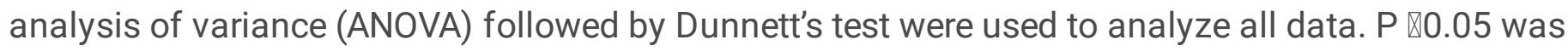
considered to be statistically significant. All statistical analysis was performed with the SPSS 22.0 software (IBM, NY).

\section{Results}




\subsection{Melatonin reduced NLRP3 inflammasome-mediated pyroptosis in mice after $\mathrm{SCl}$}

As shown in Fig. 1A, all mice were randomly divided into three groups (Sham, $\mathrm{SCl}$ and $\mathrm{SCl}+\mathrm{MT}$ ). 10 mice in each group were sacrificed for histological analysis at 3 days post-injury and the other 10 mice were used to assess the functional recovery using BMS score system at 1, 3, 7, 14, 21, 28 days post-SCl. We can easily see in Fig. 1B that higher BMS scores were exhibited in SCI-MT group compared to SCI group at each time points after SCl. As expected, the protein level of NLRP3 inflammasome and pyroptosisrelated proteins (including NLRP3, GSDMD, GSDMD-NT, Cleaved Caspase-1) were upregulated obviously in the $\mathrm{SCl}$ group as demonstrated by the western blot (WB) assay in Fig. 1C-D. Similarly, the level of serum $\mathrm{LDH}$ in $\mathrm{SCl}$ mice was also obviously higher than that in the mice of Sham group, which was assessed by the commercial LDH kits (Fig. 1E). In addition, the results of ELISA indicated that the level of released proinflammatory cytokines (TNF-a, IL-1 $\beta$ and IL-18) in serum were all significantly elevated after SCI (Fig. 1F). The increased expression of NLRP3 was confirmed by immunofluorescence (Fig. 1G). Notably, all of these above changes were obviously reversed by melatonin administration (10mg/kg, i.p) (Fig. 1C$\mathrm{G})$. It preliminary demonstrated that melatonin has the ability to mitigate the pyroptosis-mediated neuroinflammation and could promote the motor function recovery after SCl in mice.

\subsection{Melatonin suppressed the activation of STING-mediated signaling pathway after SCl in mice}

To investigate whether the STING-mediated signaling pathway participated in the pathological process after $\mathrm{SCl}$, we detected the expression level of STING pathway in the spinal cord samples by WB and RTqPCR assays. As indicated in Fig. 2A-C, the mRNA and protein level of STING were both increased in SCI group comparing to Sham group. And although there is no significant difference on the mRNA level of TBK1 and IRF3 among three groups, the protein phosphorylation levels of TBK1 and IRF3 (two main downstream effectors of the STING-mediated pathway) were obviously elevated in the SCl group than that in the Sham group. Moreover, the higher level of cGAMP production was detected in the $\mathrm{SCl}$ group (Fig. 2D). Importantly, the results of immunofluorescence (IF) staining in Fig. 2E showed that the STING was co-localized with Iba-1 (a microglia marker), which implied that STING pathway activation was mainly located in the microglia cell after SCI. Unsurprisingly, the IF intensity expression pattern of STING was identical to the results of WB and RT-qPCR. Of note, all the increased markers that representing the STING pathway activation in SCl group were all obviously decreased in SCl-MT group, which fully demonstrated that the melatonin could effectively blocked the activation of the STING signaling pathway after the spinal cord injury.

\subsection{C-176 attenuated neuroinflammation and promoted functional recovery of locomotion by alleviating NLRP3 inflammasome and pyroptosis after $\mathrm{SCl}$ in mice}

As mentioned above that the STING pathway activation was located in microglia, and we have reported NLRP3 inflammasome-mediated pyroptosis is also mainly occurs in microglia after SCl in our previous study[16], so we adopted the specific small-molecule inhibitor of the STING, C-176, to further clarify the 
relationship between the pyroptosis and the STING pathway in the microglia during SCI. As shown by WB (Fig. 3A), the protein level of pyroptosis-related proteins (NLRP3, GSDMD, GSDMD-NT and Cleaved Caspase-1) were all significantly inhibited by $\mathrm{C}-176$ after SCl. Furthermore, the increased LDH level and the pro-inflammatory cytokines (TNF-a, IL-1 $\beta$ and IL-18) after SCI were all attenuated by C-176, which were assessed by commercial kits, respectively (Fig. 3B-C). The higher BMS score (Fig. 3D) in the SCl + C176 group indicated that mice recovered faster with the $\mathrm{C}-176$ treatment. What's more, the HE staining presented the reduced inflammatory response after SCl in the presence of $\mathrm{C}-176$ (Fig. 3E). The more Nissl body in Nissl staining in C-176 group also proved that C-176 could attenuated neuronal loss (Fig. 3E). Additionally, the negative effect of $\mathrm{C}-176$ on the pyroptosis after SCl was further illustrated $\mathrm{d}$ by the results of TUNEL staining as well (Fig. 3F). In short, these above results fully revealed that C-176 exerted its positive role on $\mathrm{SCl}$ depending on inhibiting the NLRP3 inflammasome-induced pyroptosis, which greatly indicated that the STING-mediated signaling pathway likely lies in the upstream of the NLRP3 inflammasome-mediated pyroptosis after SCl.

\subsection{Overexpression of STING abolished the anti-pyroptotic effects of melatonin in LPS/ATP-induced BV2 cells}

The BV2 cell (a type of microglia cell line) were used to further elucidate the relationship between NLRP3 inflammasome-mediated pyroptosis and the STING signaling pathway in vitro. To simulate the microenvironment during spinal cord injury, the LPS $(1000 \mathrm{ng} / \mathrm{ml}, 4 \mathrm{~h})$ and ATP $(5 \mathrm{mM}, 2 \mathrm{~h})$ were used in combination to establish a cell pyroptosis model in vitro. Firstly, to determine the appropriate intervention concentration, the BV2 cells were treated with a series concentration of MT $(5,10100,500$ and $1000 \mu M)$. The results of cell viability (Fig. 4A) showed that 5 and $10 \mu \mathrm{M}$ could be more appropriate. In Fig. 4B, the typical morphological features of pyroptosis (cell swelling, prominent bubble-like vesicles as indicated by red arrows) was obvious under the light microscope in LPS/ATP group. To further confirm pyroptosis, we detected the key pyroptotic proteins (NLRP3, GSDMD, GSDMD-NT, and Cleaved caspase-1) by WB assay. Immunoblot results revealed that all of these proteins increased significantly in LPS/ATP-treated group (Fig. 4C). Also, the markedly increased level of LDH and the pro-inflammatory cytokines (TNF-a, IL-1 $\beta$, IL18 ) in the cell culture supernatant, as well as the higher PI-positive cell ratio also confirmed the loss of cell membrane integrity (Fig. 4D-E).

To verify the anti-pyroptotic role of melatonin in vitro, the BV2 cells were pre-treated with melatonin $(5 \mu \mathrm{M}$ and $10 \mu \mathrm{M}$ ) for $24 \mathrm{~h}$ prior to the LPS/ATP stimulation. As evidenced by the results in Fig. 4B-E, the BV2 cell pyroptosis was successfully suppressed by melatonin $(5 \mu \mathrm{M}$ and $10 \mu \mathrm{M})$.

Besides, the activated STING signaling pathway (increased STING-associated protein and higher cGMP level) was also blocked by melatonin as presented in Fig. 5A-B. Other than that, the lower fluorescence intensity of STING and IRF-3 in MT pre-treated group $(5 \mu \mathrm{M}$ and $10 \mu \mathrm{M})$ in the IF staining results also corroborating the above results (Fig. 5C-D).

Then to test if the melatonin played its role depending on STING, the level of STING in BV2 cells was upregulated by adenovirus. Intriguingly, the Ad-STING greatly reversed the anti-pyroptotic role of 
melatonin in LPS/ATP-induced BV2 cells (Fig. 6A). Consequently, the level of LDH and the proinflammatory cytokines (TNF-a, IL-1 $\beta, I L-6$ ) all showed the similar change trend to the results of WB (Fig. 6B-C). In addition, the results of PI staining (Fig. 6D) further demonstrated that STING overexpression reversed the anti-pyroptotic effects of melatonin. Also, the IF staining of NLRP3 showed the weaker fluorescence intensity with the pre-treatment of melatonin, while the intensity increased again as the STING were upregulated (Fig. 6E). Overall, the results above jointly demonstrated that melatonin alleviated the LPS/ATP-induced pyroptosis in a STING-dependent way in BV2 cells.

\subsection{Melatonin suppressed STING pathway by reducing ROS production and mtDNA release in LPS/ATP exposed BV2 cells}

ROS overproduction triggers the release of cytosolic mitochondrial DNA, which has been reported to be associated with STING signaling pathway activation. Therefore, we sought to identify the possible mechanism on how the melatonin regulating the STING/NLRP3 pathway. A DCFH-DA fluorescence probe was used to measure the production of ROS in LPS/ATP-induced BV2 cells. The representative fluorescence image in Fig. 7A showed that LPS/ATP provoked a robust increase of ROS production in BV2 cells. Importantly, the relative fluorescence intensity of ROS was significantly suppressed by melatonin $(5 \mu \mathrm{M}$ and $10 \mu \mathrm{M})$. Correspondently, the higher level of cytosolic mtDNA in LPS/ATP group were all decreased greatly in melatonin pre-treatment groups $(5 \mu \mathrm{M}$ and $10 \mu \mathrm{M})$. Taken together, these results implied that melatonin suppressed the activation of STING/NLLR3 pathway via decreasing the production of ROS and the release of cytosolic mtDNA.

\section{Discussion}

In this study, we proved the anti-pyroptotic effect of melatonin on $\mathrm{SCl}$, and initially identify the role of STING/NLRP3 signal axis in the pharmacological process of melatonin after SCl.

At present, spinal cord injury (SCl) is still one of the most catastrophic neurological injury diseases and no effective therapies yet are available clinically. Nowadays, a large amount evidence has shown that microglia-mediated neuroinflammation is responsible for the development of $S C I[6,41,42]$. Therefore, attenuating neuroinflammation would be a prospective strategy for treating $\mathrm{SCl}$. Based on many previous researches, melatonin seems to be a promising candidate to treat $\mathrm{SCl}$ for its various therapeutic effects including lowering edema[43], inhibiting scar formation[44], attenuating microgliosis[45]. In addition, Zhang $Y$ [46] reported that melatonin could reduce neuroinflammation by promoting M2 microglia polarization, subsequently improved functional recovery after $\mathrm{SCl}$. Besides, the microglia pyroptosis has also emerged as another important resource of neuroinflammation in $\mathrm{SCl}$. In our previous studies, we have identified the crucial role of microglia pyroptosis in SCI[15]. It is a novel kind of inflammatory programmed cell death characterized by cell swelling and lysis, leading to the secretion of proinflammatory cytokines (including IL-1 $\beta$, IL-18 and TNF-a). As we know, inflammasomes activation is the crucial initial event to mediate the pyroptosis. And in general, five kinds of inflammasomes (NLRP1, NLRC4, AIM2, RIG-1 and NLRP3) has been identified while the NLRP3 inflammasome is the most widely 
investigated in SCl. However, few studies focused on exploring whether melatonin exerts its regulatory role through regulating the NLRP3 inflammasome after SCI. And no reports are published about the role of STING pathway on regulating NLRP3 inflammasome during the pathological process after SCI.

In the present study, we found that melatonin effectively inhibited the expression of NLRP3 inflammasome associated proteins: NLRP3, ASC, Cleaved Caspase-1 and GSDMD (the executor of pyroptosis), which were all greatly increased after SCI. Moreover, it is notable that the protein level of STING and its downstream molecule (P-TBK1 and P-IRF3) were also upregulated after SCI. The STINGmediated pathway, as a part of innate immune system, has been found to play a significant role in modulating infection and inflammation $[47,48]$. Increasing evidences have indicated the regulating role of STING signaling pathway in neuroinflammation and neurological diseases[49-51]. Sliter [52] showed that STING deficiency eliminated inflammatory phenotypes in Parkin- and PINK1-deficient mice. Jauhari A [37] also reported the increased level of cytosolic DNA and inflammation with the activated cGAS/STING/IRF3 in HD patients. And to our knowledge, it is the first time that STING-mediated pathway participated in the $\mathrm{SCl}$ has been reported. Importantly, our results in vivo and in vitro together indicated that melatonin effectively blocked the activation of STING signaling pathway after SCl.

In recent years, the interplay between STING and NLRP3 inflammasome has been gradually revealed. In an LPS-induced cardiac injury model, the NLRP3 inflammasome was activated by the stimulation of STING, and the protection role of STING knockdown was reversed by NLRP3 overexpression[24]. What's more, Li N[25] also proved that STING deficiency inhibited NLRP3 inflammasome and pyroptosis in LPStreated Lung. Here, we also discovered that downregulation of STING by C-176 obviously reduced the NLRP3 inflammasome activation in vivo. On the other hand, overexpression of STING abolished the antipyroptotic effect of melatonin in LPS/ATP treated BV2 cells. These results are consistent with the previous published studies and together indicated that the anti-pyroptotic role of melatonin is mediated by the blockage of STING-mediated pathway.

It is well-known that NLRP3 inflammasome activation is related to multiple signal molecules including DAMPs, oxidant stress, mitochondrial dysfunction as well as reactive oxygen species (ROS) [53-55]. Wang BR[56] demonstrated that ROS was essential in PM2.5-induced IL-1 $\beta$ production and NLRP3 inflammasome activation in oA $\beta$-stimulated microglia. What's more, ROS-mediated the release of mtDNA into cytoplasm has been reported to be responsible for the activation of cGAS/STING pathway[57]. On the other hand, it is also acknowledged that melatonin plays a crucial role in multiple diseases as a mitochondria-targeted antioxidant[58, 59]. Hence, we speculated melatonin suppressed STING/NLRP3 axis by alleviating oxidative stress-related factors. In agreement with the previous published studies, we found that the increased ROS and cytosolic mIDNA was detected in LPS/ATP induced microglia pyroptosis. And the reduced ROS production and mtDNA release in the melatonin pre-treatment groups demonstrated that melatonin exerts its protection role by reducing pyroptotic neuroinflammation via blocking the ROS/mtDNA-activated STING/NLRP3 signaling axis. Of course, a shortcoming in our study is the lack of STING knock-out mice, it would provide more powerful evidence to our conclusion. In 
addition, the detail molecular mechanism of the activation of STING-mediated pathway in SCI is worth exploring in our further studies.

\section{Conclusion}

In conclusion, as depicted in Fig. 8, we reported for the first time that the ROS/mtDNA/STING pathway undertook an important role in NLRP3 inflammasome-mediated pyroptosis after SCl in mice. It was also a novel mechanism that interpreting the anti-pyroptotic effect of melatonin on $\mathrm{SCl}$ and thus provided potential therapeutic target for treating SCl.

\section{Abbreviations}

SCl: Spinal cord injury;

MT: Melatonin;

PAMPs: Pathogen-associated molecular patterns;

DAMPs: Damage-associated molecular patterns;

cGAS: Cyclic GMP-AMP synthase;

STING: Stimulator of interferon genes;

GSDMD: Gasdermin D;

NLRP3: NLR family pyrin domain-containing-3;

ASC: Apoptosis-associated speck-like protein;

i.p.: Intraperitoneal injection;

CCK-8: Cell Counting Kit-8;

PI: Propidium lodide;

WB: Western Blot;

RT-qPCR: real-time quantitative polymerase chain reaction;

ELISA: Enzyme-linked immune-sorbent assay;

H\&E: Hematoxylin and eosin;

ROS: Reactive oxygen species. 


\section{Declarations}

\section{Acknowledgements}

The authors thank Prof. PingZhou (Department of Physiology and Pathophysiology, School of Basic Medical Sciences, Fudan University) for providing the experimental equipment, valuable suggestions and technical assistance during this study.

\section{Authors' contributions}

All authors read and approved the final manuscript. Jin Wang, Haiyuan Yang and Fan Zhang performed the experiments and drafted the manuscript; Jin Wang, Fan Zhang and Minghao Shao conducted the data analysis; Haiyuan Yang and Haocheng Xu reviewed and edited the manuscript. Shun Xu and Feizhou Lyu conceived and designed the study. The authors read and approved the final manuscript.

\section{Availability of data and materials}

The datasets used and/or analyzed during the current study are available from the corresponding author on reasonable request.

\section{Funding}

This work was supported by the National Natural Science Foundation of China (grant number 81472056) and Shanghai Sailing Program (grant number: 20YF1438100).

\section{Ethics Declarations}

Ethics approval and consent to participate

All animal experiments were approved by the Ethics Committee of Experimental Research, Huashan Hospital, Fudan University (Shanghai, China).

\section{Consent for publication}

Not applicable.

\section{Competing interests}

The authors have declared that no competing interests exist.

\section{Author details}

Affiliations

Department of Huashan Hospital, Fudan Universtiy, Shanghai 200040, China 
Jin Wang, Haiyuan Yang, Fan Zhang, Minghao Shao, Haocheng Xu, Feizhou Lyu

Department of Orthopedics, Shanghai Fifth People's Hospital, Fudan University, Shanghai 200240, China

Shun Xu, Feizhou Lyu

Jin Wang, Haiyuan Yang and Fan Zhang are co-first author.

Corresponding author: Shun Xu and Feizhou Lyu.

\section{References}

1. Jain, N.B., et al., Traumatic spinal cord injury in the United States, 1993-2012. JAMA, 2015. 313(22): p. 2236-43.

2. Oyinbo, C.A., Secondary injury mechanisms in traumatic spinal cord injury: a nugget of this multiply cascade. Acta Neurobiol Exp (Wars), 2011. 71(2): p. 281-99.

3. McDonald, J.W. and C. Sadowsky, Spinal-cord injury. Lancet, 2002. 359(9304): p. 417-25.

4. Karsy, M. and G. Hawryluk, Modern Medical Management of Spinal Cord Injury. Curr Neurol Neurosci Rep, 2019. 19(9): p. 65.

5. Xu, S., et al., Ecto-5'-nucleotidase (CD73) attenuates inflammation after spinal cord injury by promoting macrophages/microglia M2 polarization in mice. J Neuroinflammation, 2018. 15(1): p. 155.

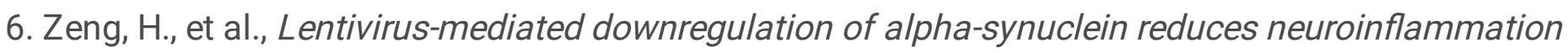
and promotes functional recovery in rats with spinal cord injury. J Neuroinflammation, 2019. 16(1): p. 283.

7. Davis, B.K., H. Wen, and J.P. Ting, The inflammasome NLRs in immunity, inflammation, and associated diseases. Annu Rev Immunol, 2011. 29: p. 707-35.

8. Jo, E.K., et al., Molecular mechanisms regulating NLRP3 inflammasome activation. Cell Mol Immunol, 2016. 13(2): p. 148-59.

9. He, Y., H. Hara, and G. Nunez, Mechanism and Regulation of NLRP3 Inflammasome Activation. Trends Biochem Sci, 2016. 41(12): p. 1012-1021.

10. Jia, Y., et al., Metformin protects against intestinal ischemia-reperfusion injury and cell pyroptosis via TXNIP-NLRP3-GSDMD pathway. Redox Biol, 2020. 32: p. 101534.

11. Tong, Y., et al., NLRP3 Inflammasome and Its Central Role in the Cardiovascular Diseases. Oxid Med Cell Longev, 2020. 2020: p. 4293206.

12. Pavillard, L.E., et al., Cardiovascular diseases, NLRP3 inflammasome, and western dietary patterns. Pharmacol Res, 2018. 131: p. 44-50.

13. Chang, Y., et al., NLRP3 inflammasome-mediated microglial pyroptosis is critically involved in the development of post-cardiac arrest brain injury. J Neuroinflammation, 2020. 17(1): p. 219. 
14. Youm, Y.H., et al., Canonical NIrp3 inflammasome links systemic low-grade inflammation to functional decline in aging. Cell Metab, 2013. 18(4): p. 519-32.

15. Xu, S., et al., CD73 alleviates GSDMD-mediated microglia pyroptosis in spinal cord injury through PI3K/AKT/Foxo1 signaling. Clin Transl Med, 2021. 11(1): p. e269.

16. Xu, S., et al., TLR4 promotes microglial pyroptosis via IncRNA-F630028010Rik by activating PI3K/AKT pathway after spinal cord injury. Cell Death Dis, 2020. 11(8): p. 693.

17. Al Mamun, A., et al., Role of pyroptosis in spinal cord injury and its therapeutic implications. J Adv Res, 2021. 28: p. 97-109.

18. Hopfner, K.P. and V. Hornung, Molecular mechanisms and cellular functions of cGAS-STING signalling. Nat Rev Mol Cell Biol, 2020. 21(9): p. 501-521.

19. Wan, D., W. Jiang, and J. Hao, Research Advances in How the cGAS-STING Pathway Controls the Cellular Inflammatory Response. Front Immunol, 2020. 11: p. 615.

20. Chen, Q., L. Sun, and Z.J. Chen, Regulation and function of the cGAS-STING pathway of cytosolic DNA sensing. Nat Immunol, 2016. 17(10): p. 1142-9.

21. Motwani, M., S. Pesiridis, and K.A. Fitzgerald, DNA sensing by the CGAS-STING pathway in health and disease. Nat Rev Genet, 2019. 20(11): p. 657-674.

22. Wang, W., et al., STING promotes NLRP3 localization in ER and facilitates NLRP3 deubiquitination to activate the inflammasome upon HSV-1 infection. PLoS Pathog, 2020. 16(3): p. e1008335.

23. Tian, Y., et al., Epigallocatechin-3-Gallate Protects H2O2-Induced Nucleus Pulposus Cell Apoptosis and Inflammation by Inhibiting cGAS/Sting/NLRP3 Activation. Drug Des Devel Ther, 2020. 14: p. 2113-2122.

24. Li, N., et al., STING-IRF3 contributes to lipopolysaccharide-induced cardiac dysfunction, inflammation, apoptosis and pyroptosis by activating NLRP3. Redox Biol, 2019. 24: p. 101215.

25. Ning, L., et al., Cytosolic DNA-STING-NLRP3 axis is involved in murine acute lung injury induced by lipopolysaccharide. Clin Transl Med, 2020. 10(7): p. e228.

26. Srinivasan, V., et al., Therapeutic actions of melatonin in cancer: possible mechanisms. Integr Cancer Ther, 2008. 7(3): p. 189-203.

27. Najafi, M., et al., The melatonin immunomodulatory actions in radiotherapy. Biophys Rev, 2017. 9(2): p. 139-148.

28. Li, T., et al., Exogenous melatonin as a treatment for secondary sleep disorders: A systematic review and meta-analysis. Front Neuroendocrinol, 2019. 52: p. 22-28.

29. Xie, Z., et al., A review of sleep disorders and melatonin. Neurol Res, 2017. 39(6): p. 559-565.

30. Abdel Moneim, A.E., et al., Protective effects of melatonin against oxidative damage induced by Egyptian cobra (Naja haje) crude venom in rats. Acta Trop, 2015. 143: p. 58-65.

31. Cipolla-Neto, J. and F.G.D. Amaral, Melatonin as a Hormone: New Physiological and Clinical Insights. Endocr Rev, 2018. 39(6): p. 990-1028. 
32. Arioz, B.I., et al., Melatonin Attenuates LPS-Induced Acute Depressive-Like Behaviors and Microglial NLRP3 Inflammasome Activation Through the SIRT1/Nrf2 Pathway. Front Immunol, 2019. 10: p. 1511.

33. Che, $\mathrm{H}$., et al., Melatonin alleviates cardiac fibrosis via inhibiting IncRNA MALAT1/miR-141-mediated NLRP3 inflammasome and TGF-beta1/Smads signaling in diabetic cardiomyopathy. FASEB J, 2020. 34(4): p. 5282-5298.

34. Zhang, Y., et al., Melatonin alleviates acute lung injury through inhibiting the NLRP3 inflammasome. J Pineal Res, 2016. 60(4): p. 405-14.

35. Liu, Z., et al., Melatonin alleviates inflammasome-induced pyroptosis through inhibiting NFkappaB/GSDMD signal in mice adipose tissue. J Pineal Res, 2017. 63(1).

36. Zhang, Y., et al., Melatonin prevents endothelial cell pyroptosis via regulation of long noncoding RNA MEG3/miR-223/NLRP3 axis. J Pineal Res, 2018. 64(2).

37. Jauhari, A., et al., Melatonin inhibits cytosolic mitochondrial DNA-induced neuroinflammatory signaling in accelerated aging and neurodegeneration. J Clin Invest, 2020. 130(6): p. 3124-3136.

38. Haag, S.M., et al., Targeting STING with covalent small-molecule inhibitors. Nature, 2018. 559(7713): p. 269-273.

39. Basso, D.M., et al., Basso Mouse Scale for locomotion detects differences in recovery after spinal cord injury in five common mouse strains. J Neurotrauma, 2006. 23(5): p. 635-59.

40. West, A.P., et al., Mitochondrial DNA stress primes the antiviral innate immune response. Nature, 2015. 520(7548): p. 553-7.

41. Liu, Z., et al., Advanced oxidation protein products induce microglia-mediated neuroinflammation via MAPKs-NF-kappaB signaling pathway and pyroptosis after secondary spinal cord injury. J Neuroinflammation, 2020. 17(1): p. 90.

42. Chen, J., et al., Neuron and microglia/macrophage-derived FGF10 activate neuronal FGFR2/PI3K/Akt signaling and inhibit microglia/macrophages TLR4/NF-kappaB-dependent neuroinflammation to improve functional recovery after spinal cord injury. Cell Death Dis, 2017. 8(10): p. e3090.

43. Li, C., et al., Melatonin lowers edema after spinal cord injury. Neural Regen Res, 2014. 9(24): p. 220510.

44. Krityakiarana, W., et al., Effects of melatonin on severe crush spinal cord injury-induced reactive astrocyte and scar formation. J Neurosci Res, 2016. 94(12): p. 1451-1459.

45. Yang, Z., et al., Melatonin exerts neuroprotective effects by attenuating astro- and microgliosis and suppressing inflammatory response following spinal cord injury. Neuropeptides, 2020. 79: p. 102002.

46. Zhang, Y., et al., Melatonin improves functional recovery in female rats after acute spinal cord injury by modulating polarization of spinal microglial/macrophages. J Neurosci Res, 2019. 97(7): p. 733743.

47. Barber, G.N., STING: infection, inflammation and cancer. Nat Rev Immunol, 2015. 15(12): p. $760-70$. 
48. Kwon, J. and S.F. Bakhoum, The Cytosolic DNA-Sensing cGAS-STING Pathway in Cancer. Cancer Discov, 2020. 10(1): p. 26-39.

49. Chin, A.C., Neuroinflammation and the cGAS-STING pathway. J Neurophysiol, 2019. 121(4): p. 10871091.

50. Paul, B.D., S.H. Snyder, and V.A. Bohr, Signaling by cGAS-STING in Neurodegeneration, Neuroinflammation, and Aging. Trends Neurosci, 2021. 44(2): p. 83-96.

51. Mathur, V., et al., Activation of the STING-Dependent Type I Interferon Response Reduces Microglial Reactivity and Neuroinflammation. Neuron, 2017. 96(6): p. 1290-1302 e6.

52. Sliter, D.A., et al., Parkin and PINK1 mitigate STING-induced inflammation. Nature, 2018. 561(7722): p. 258-262.

53. Zhou, R., et al., A role for mitochondria in NLRP3 inflammasome activation. Nature, 2011. 469(7329): p. 221-5.

54. Lin, Q., et al., PINK1-parkin pathway of mitophagy protects against contrast-induced acute kidney injury via decreasing mitochondrial ROS and NLRP3 inflammasome activation. Redox Biol, 2019. 26: p. 101254.

55. Gong, Z., et al., Mitochondrial dysfunction induces NLRP3 inflammasome activation during cerebral ischemia/reperfusion injury. J Neuroinflammation, 2018. 15(1): p. 242.

56. Wang, B.R., et al., PM2.5 exposure aggravates oligomeric amyloid beta-induced neuronal injury and promotes NLRP3 inflammasome activation in an in vitro model of Alzheimer's disease. J Neuroinflammation, 2018. 15(1): p. 132.

57. Cheng, A.N., et al., Mitochondrial Lon-induced mtDNA leakage contributes to PD-L 1-mediated immunoescape via STING-IFN signaling and extracellular vesicles. J Immunother Cancer, 2020. 8(2).

58. Galano, A., et al., Melatonin and its metabolites as copper chelating agents and their role in inhibiting oxidative stress: a physicochemical analysis. J Pineal Res, 2015. 58(1): p. 107-16.

59. Rodriguez, C., et al., Regulation of antioxidant enzymes: a significant role for melatonin. J Pineal Res, 2004. 36(1): p. 1-9.

\section{Tables}

Table. 1 Primers used for qRT-PCR in this study.

\begin{tabular}{lll} 
Target genes & Forward primer & Reverse primer \\
\hline STING & CCGAAGACTGTACATCCTCTTT & AGCATATCTCGGAATCGAATGT \\
\hline TBK1 & ACTGGTGATCTCTATGCTGTCA & TTCTGGAAGTCCATACGCATTG \\
\hline IRF3 & AGGCTTCTGGGCCTTATGTG & TGCTTCTCTCGCCAGGAATAC \\
\hline B-actin & GGCTGTATTCCCCTCCATCG & CCAGTTGGTAACAATGCCATGT
\end{tabular}


Figures

A
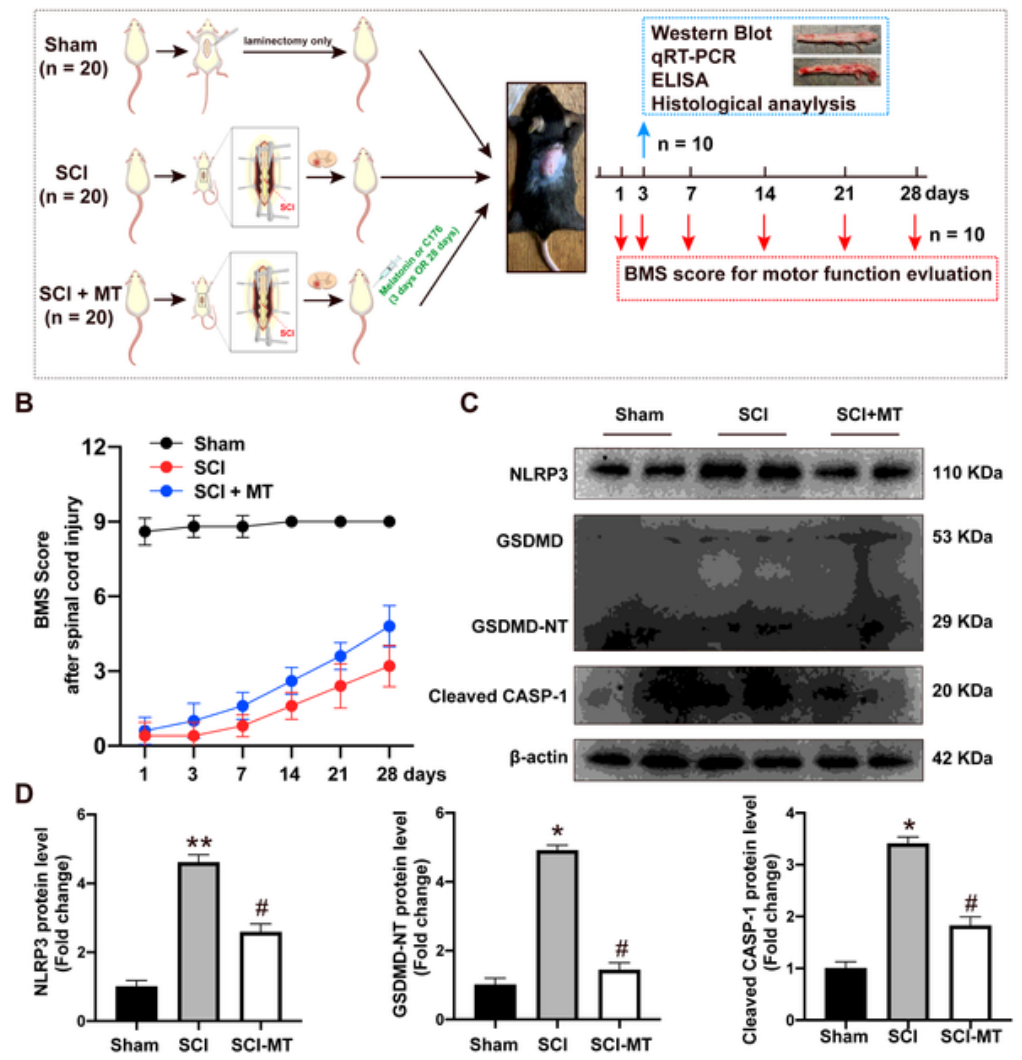

E

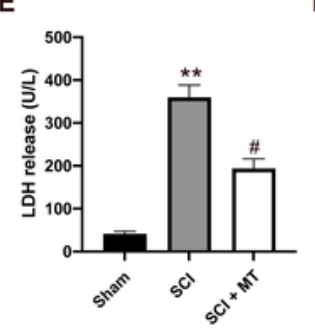

$\mathbf{F}$

G
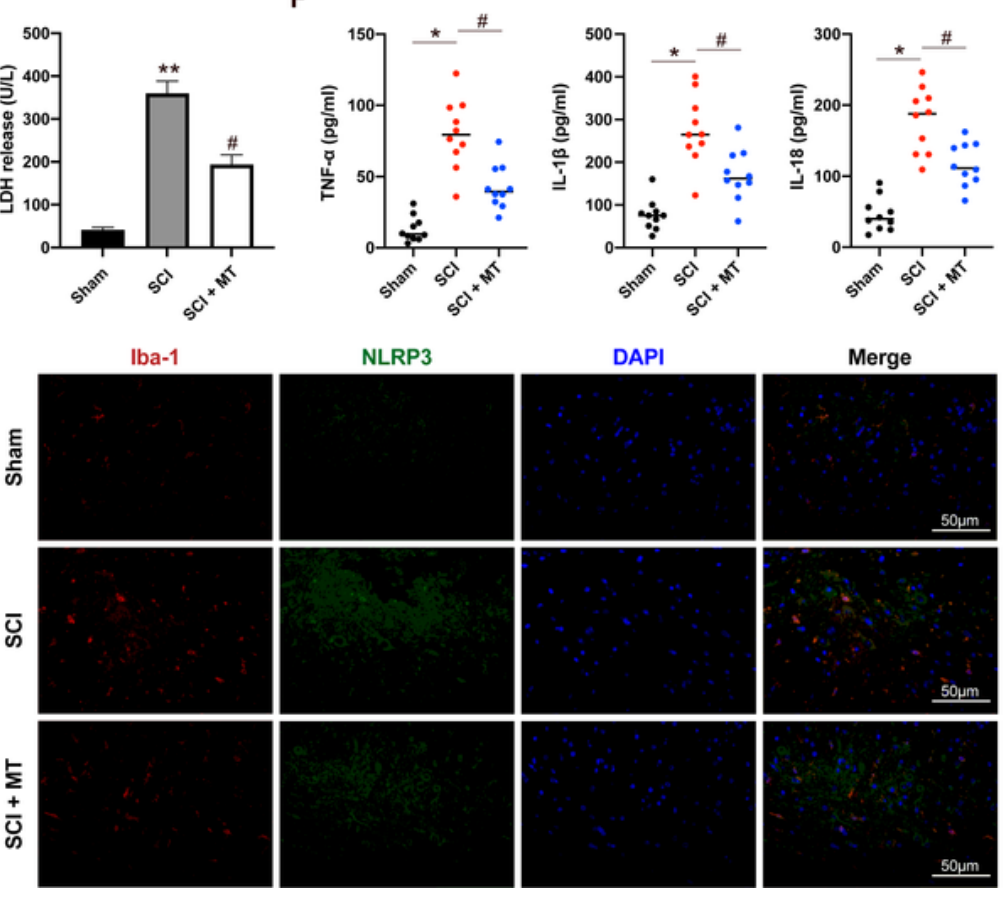

Figure 1

Melatonin inhibited the NLRP3 inflammasome-mediated pyroptosis and promoted motor function recovery after $\mathrm{SCl}$ in mice. $\mathrm{A}$. The schematic diagram of the treatment of mice in three groups (Sham, $\mathrm{SCl}$ and $\mathrm{SCl}+\mathrm{MT}$ ) and the corresponding analysis after $\mathrm{SCl}$. B. The locomotion function of mice in three 
groups was evaluated by BMS score at 1, 3, 7, 14, 21, 28 days after surgery. C-D. Representative immunoblotting and quantitative analysis of pyroptosis-associated proteins in three groups. $\mathrm{E}$. The released level of serum LDH in mice was detected by LDH Assay Kit. F. The level of serum proinflammatory cytokines (TNF- $a$, IL-1 $\beta$ and IL-18) in three groups was measured by ELISA assay. G. The representative immunofluorescence image of IBA- 1 and NLRP3 of spinal cord in three groups * $p<0.05$, ${ }^{* \star} p<0.01$ versus Sham group, $\# p<0.05$ versus SCl group. All data were shown as the mean \pm SD from 3 independent experiments.

A

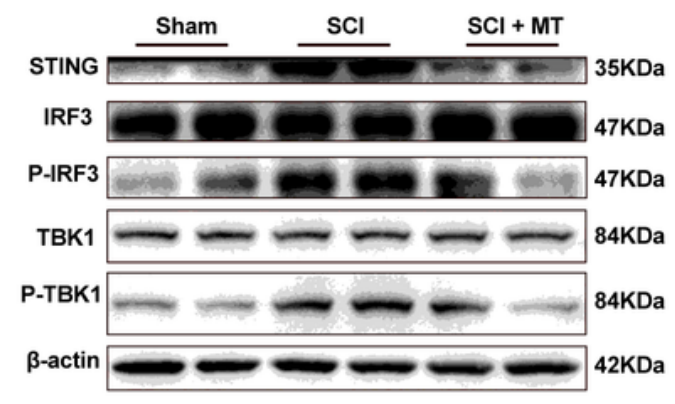

B
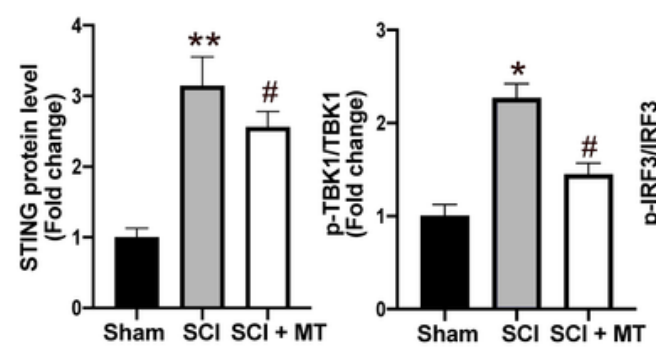

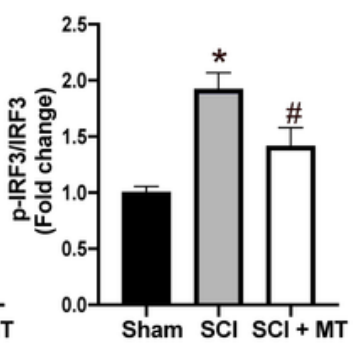

D

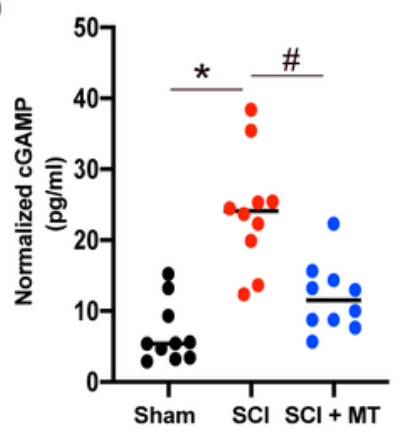

E

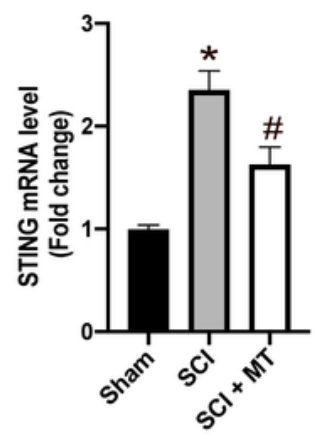

Iba-1
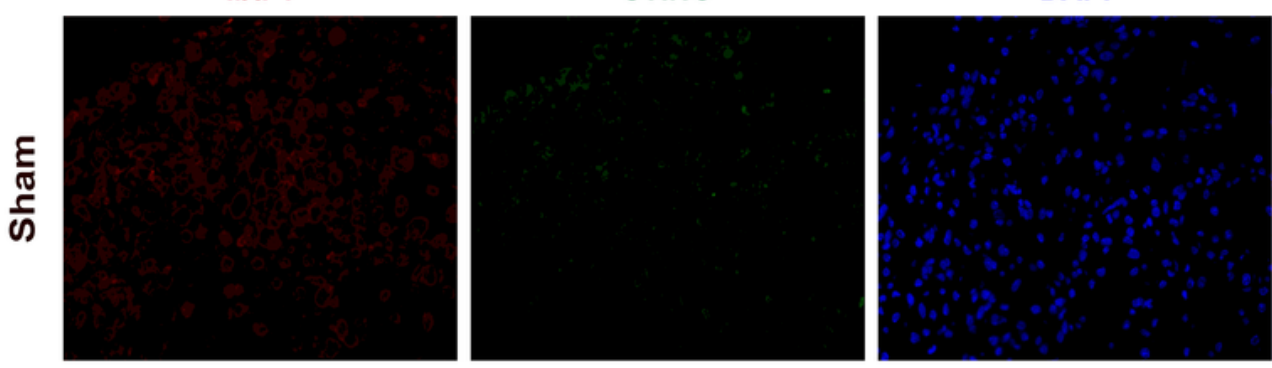

DAPI
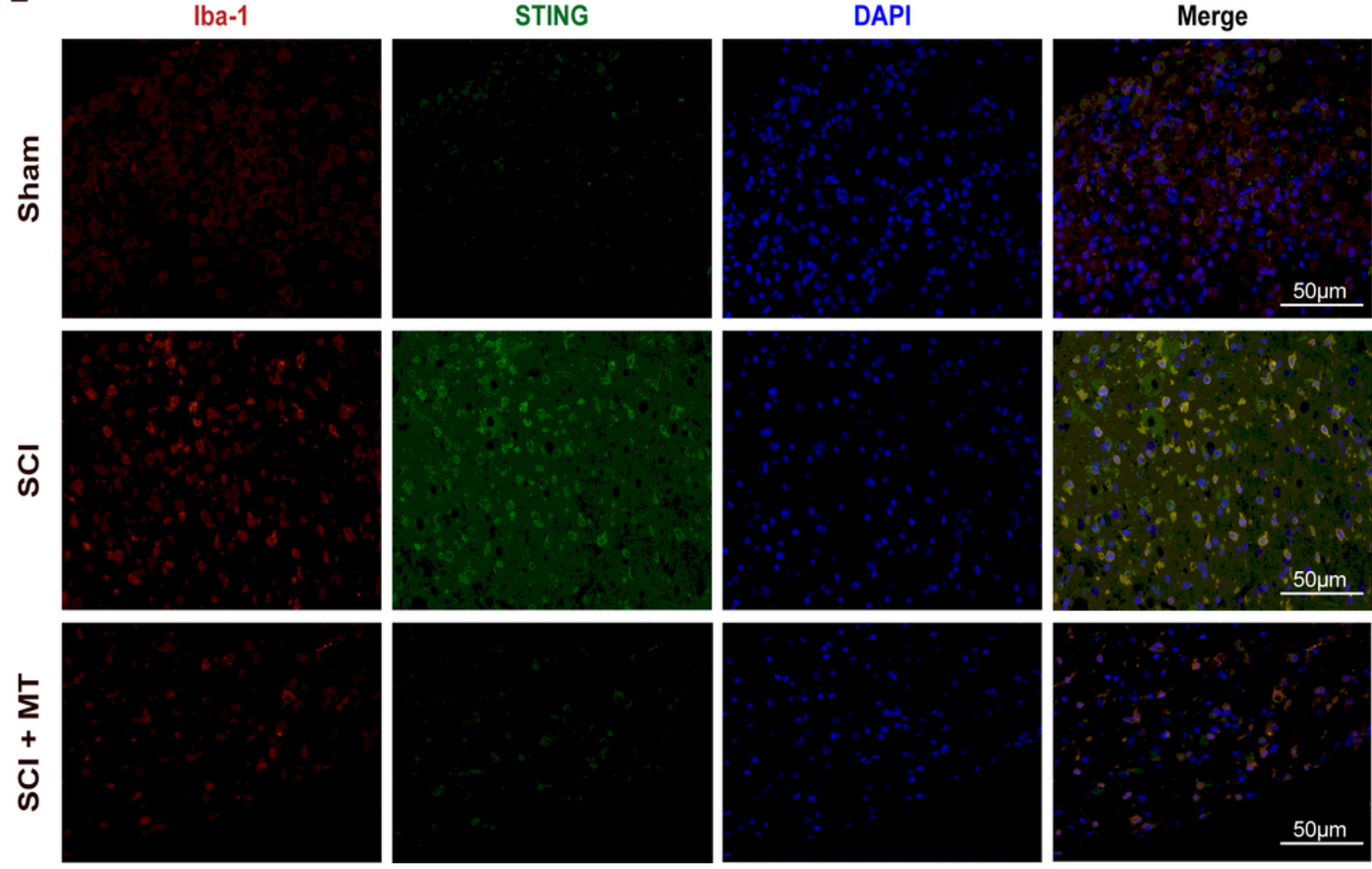

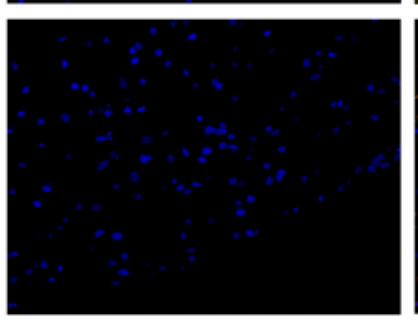




\section{Figure 2}

Melatonin impeded the activation of STING-mediated pathway after SCl in mice. A-B. Representative immunoblotting and quantitative analysis of STING pathway-associated proteins (STING, TBK1, p-TBK1, IRF3 and P-IRF3) in three groups. C. The quantitative real-time PCR results of STING, TBK1 and IRF3 in three groups. D. The level of cGAMP in three groups was measured by 2'3'-cGAMP ELISA kit. E. The representative two-photon excitation images of immunofluorescence of lba-1 (red) and STING (green) acquired from spinal cord tissue in mice at 3 days after $\mathrm{SCl}$ in three groups. Nuclei are stained with DAPI (blue). Scale bar: $50 \mu \mathrm{m} .{ }^{*} p<0.05,{ }^{\star *} p<0.01$ versus Sham group, $\# \mathrm{p}<0.05$ versus $\mathrm{SCl}$ group. All data were shown as the mean \pm SD from 3 independent experiments. 


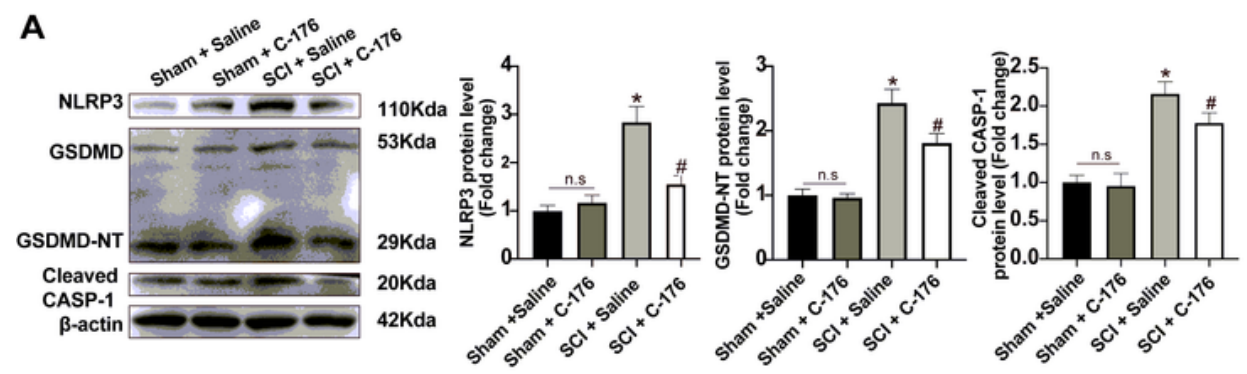

B

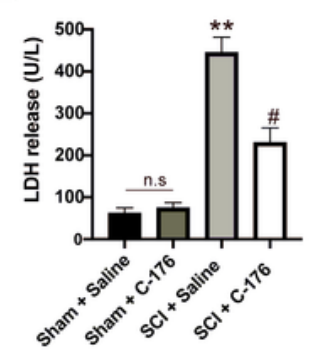

E

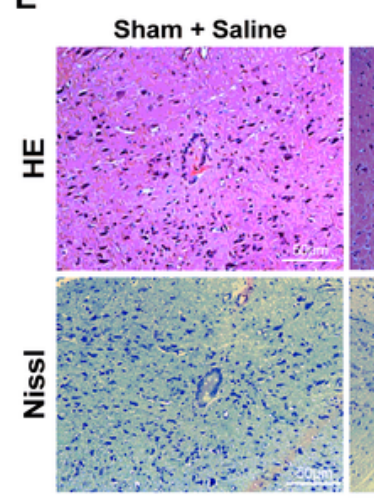

F
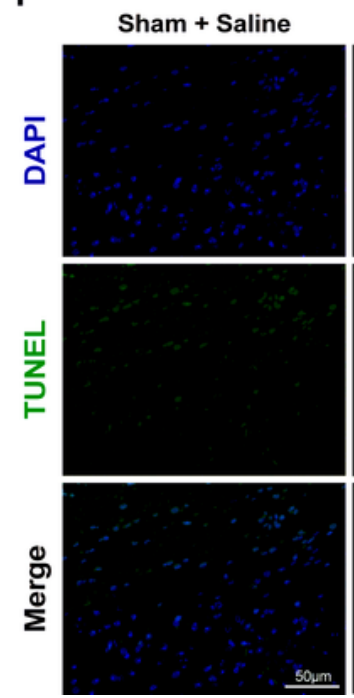

C

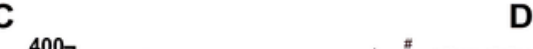

D

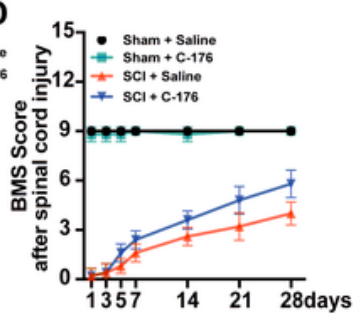

Sham + C-176

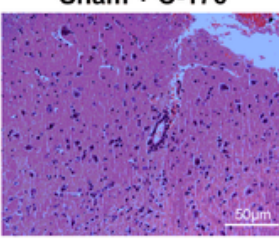

$\mathrm{SCl}+$ Saline

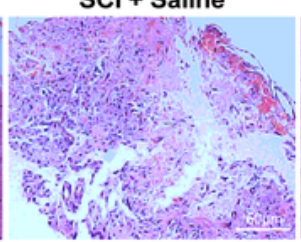

$\mathrm{SCl}+\mathrm{C}-176$

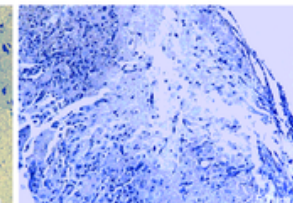

$\mathrm{SCl}+$ Saline
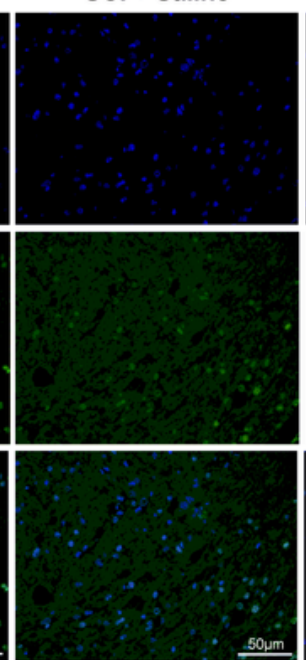

$\mathrm{SCl}+\mathrm{C}-176$

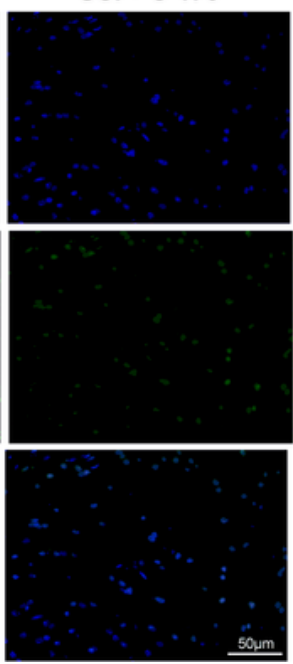

Figure 3

The STING small-molecule inhibitor C-176 alleviated NLRP3 inflammasome-induced pyroptosis and enhance locomotion recovery after $\mathrm{SCl}$ in mice. A. Representative Western blotting of STING and pyroptosis-related proteins and quantification analysis 3 days after $\mathrm{SCl}$ or Sham surgery; $\mathrm{B}$. The release level of serum LDH in different groups detected by LDH assay kits. C. The level of serum proinflammatory cytokine (TNF-a, IL-1 $\beta$ and IL-18) of mice in different groups assessed by commercial 
ELISA kits, respectively. D. The locomotion function of mice in four groups (Sham+Saline, Sham+C-176, $\mathrm{SCl}+$ Saline and $\mathrm{SCl}+\mathrm{C}-176$ ) assessed by BMS score at 1, 3, 5, 7, 14, 21,28 days post-injury. E. The representative hematoxylin-eosin (HE) and Nissl staining of different groups 3 days after $\mathrm{SCl}$ or sham

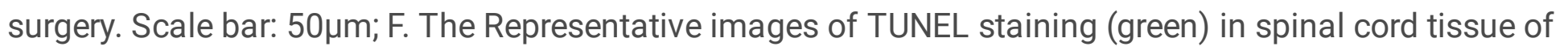
each group. Nuclei are stained with DAPI (blue). Scale bar: $50 \mu \mathrm{m}$. ${ }^{\star} p<0.05$ versus Sham+Saline group, $\# p<0.05$ versus $\mathrm{SCl}+\mathrm{Saline}$ group. All data were shown as the mean $\pm \mathrm{SD}$ from 3 independent experiments.

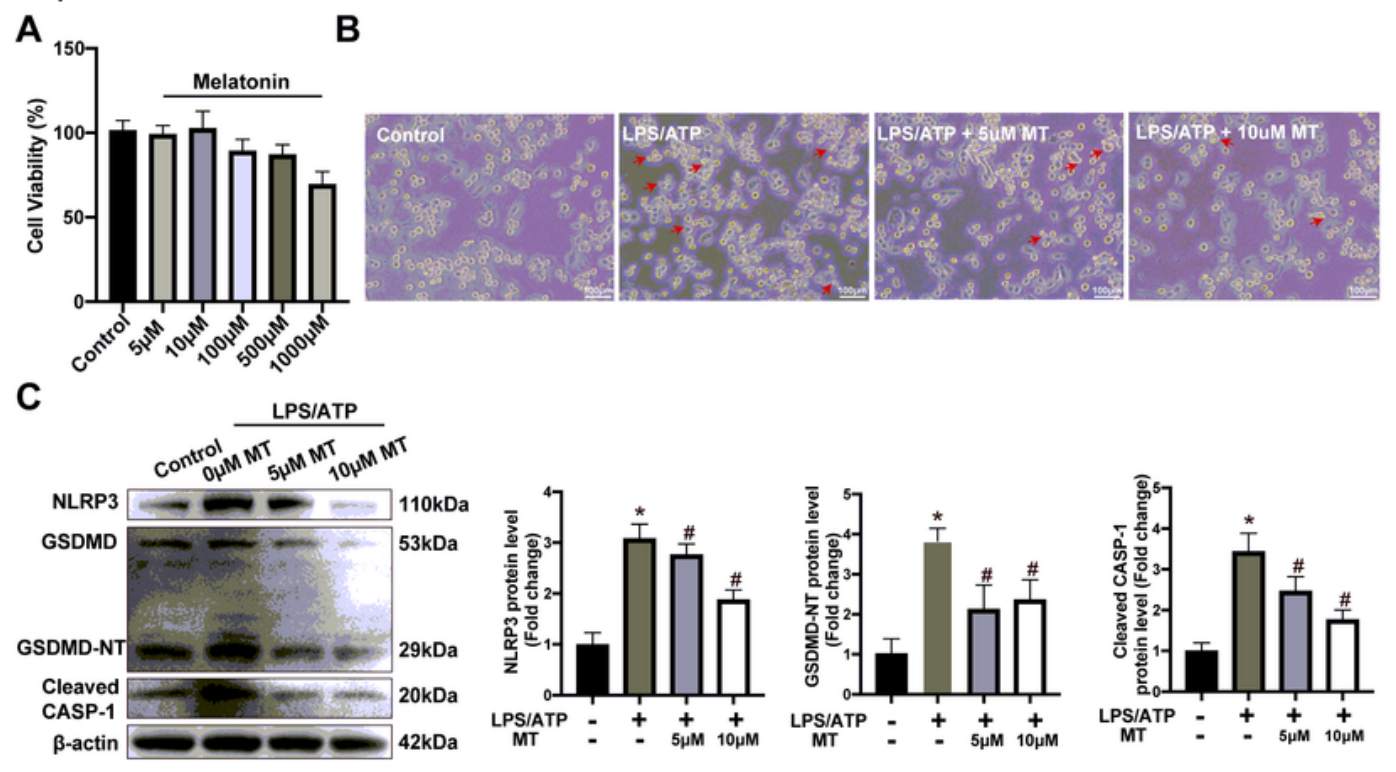

D
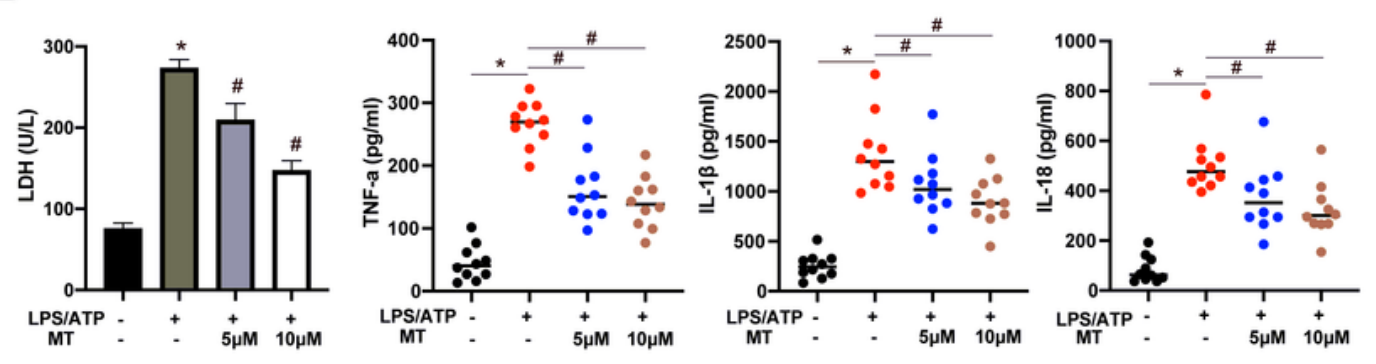

E
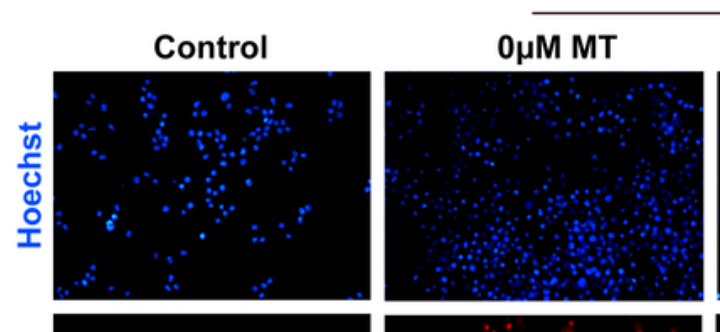

LPS/ATP
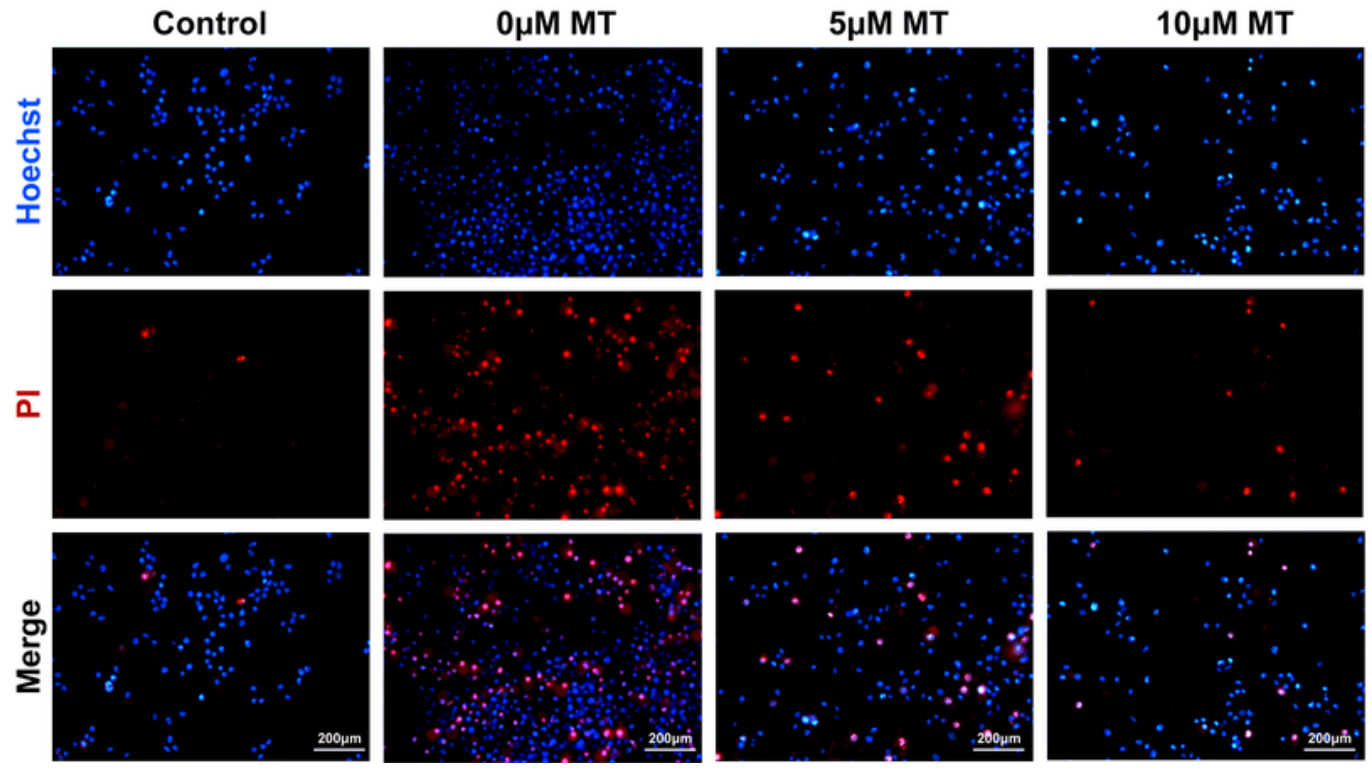


\section{Figure 4}

Melatonin inhibited the LPS/ATP induced NLRP3 inflammasome-dependent pyroptosis in BV2 cells. A. The cell viability of BV2 cells exposed to a series concentration of melatonin $(5,10,100,500$ and $1000 \mu \mathrm{M}) ; \mathrm{B}$. The representative images of cell morphology in different groups. (Cell swelling and bubblelike vesicle were indicated by red arrows), scale bar: $100 \mu \mathrm{m}$; C. The representative Western blotting of pyroptosis-associated proteins (NLRP3, GSDMD, GSDMD-NT, Cleaved Caspase-1) and quantitative analysis in different treatment groups, respectively; D. The relative LDH level and the level of serum proinflammatory cytokine (TNF-a, IL-1 $\beta$ and IL-18) in the cell culture supernatant of different groups assessed by commercial ELISA kits, respectively; E. The representative image of PI staining (red) in different groups, Nuclei were stained with Hoechst (blue), scale bar: $200 \mu \mathrm{m}$. ${ }^{*} p<0.05$ versus Control group, $\# p<0.05$ versus LPS/ATP group. All data were shown as the mean \pm SD from 3 independent experiments.

A

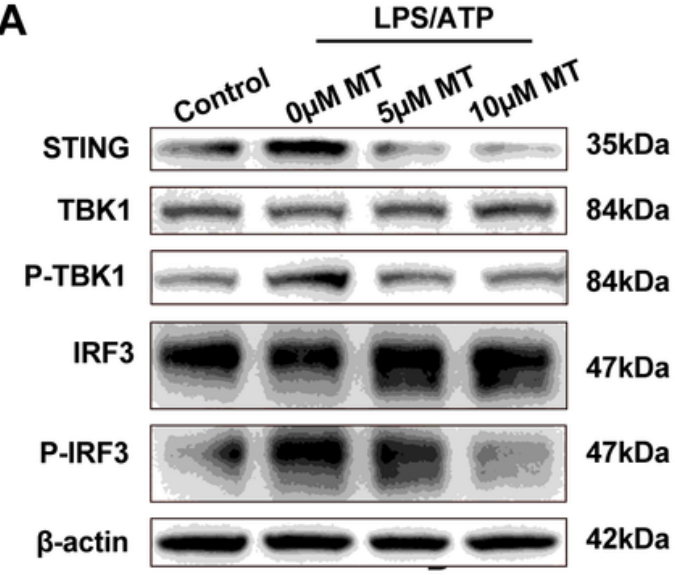

C

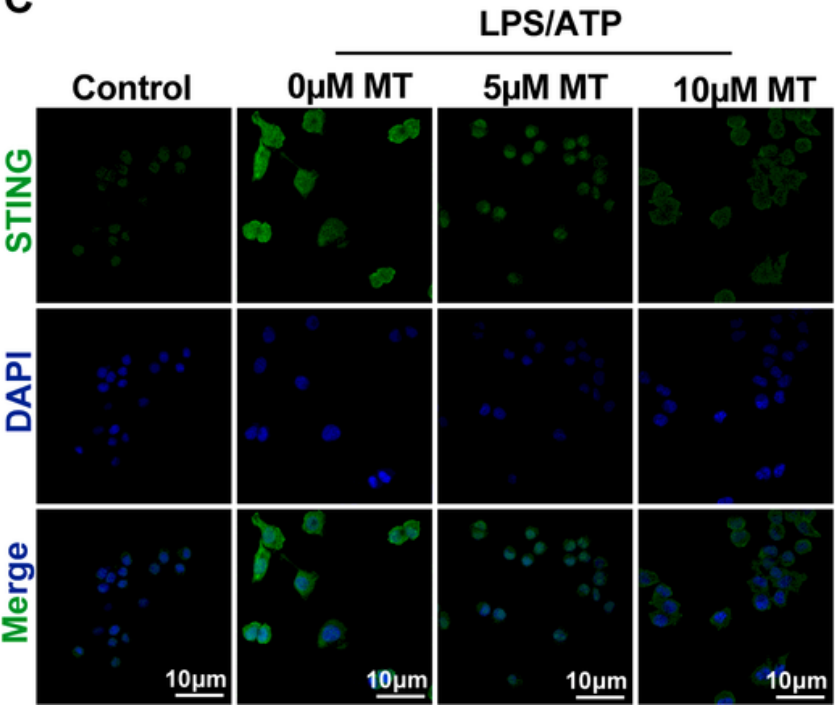

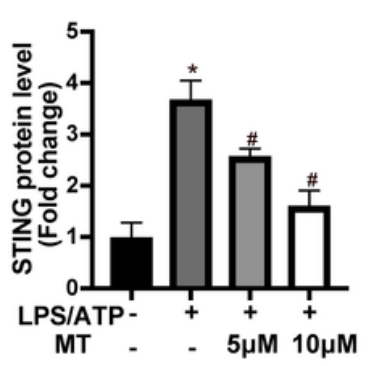
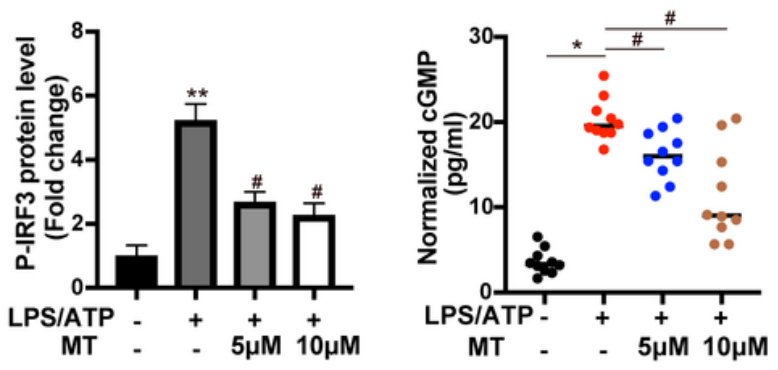

D

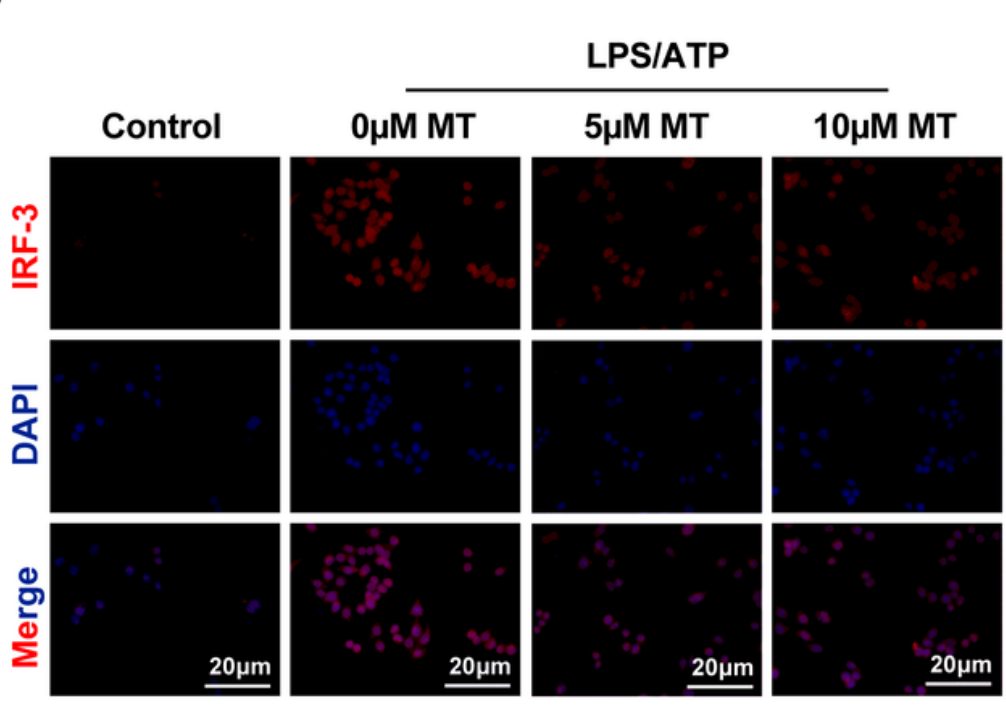

Figure 5

Melatonin effectively inhibited STING-mediated signaling pathway in LPS/ATP induced BV2 cells. A. Representative Western blotting of STING pathway-associated proteins (STING, TBK1, P-TBK1, IRF3, PIRF3) and quantitative analysis in different groups (Control, LPS/ATP, LPS/ATP+5 $\mu$ M MT and 
LPS/ATP+10 $\mu \mathrm{M} \mathrm{MT);} B$. The relative cGMP level in cell culture supernatant of four groups measured by 2'3'-cGAMP ELISA kit; C-D. The representative Immunofluorescence images of STING (E) and IRF-3 (F) in BV2 cells of different groups. Nuclei are stained with DAPI (blue). Scale bar: $10 \mu \mathrm{m}$ and $20 \mu \mathrm{m}$, respectively. ${ }^{*} p<0.05,{ }^{*} p<0.01$ versus Control group, $\# p<0.05$ versus LPS/ATP group. All data were shown as the mean \pm SD from 3 independent experiments.
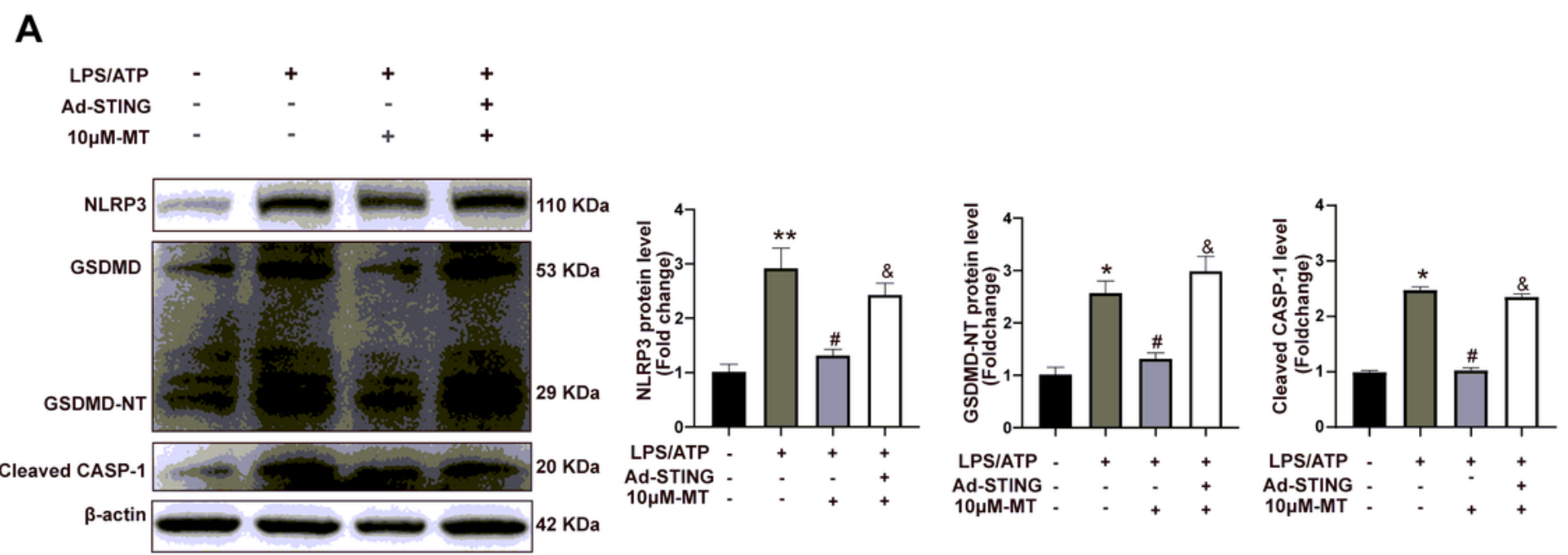

B

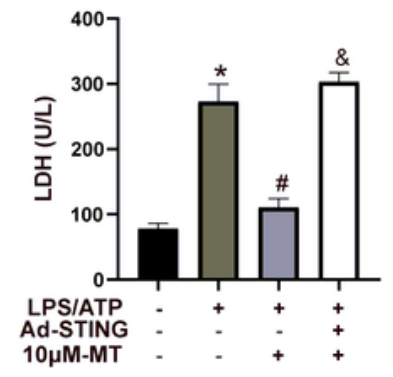

C

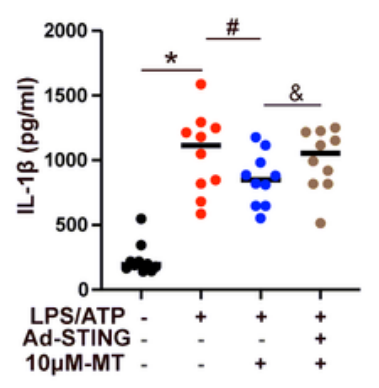

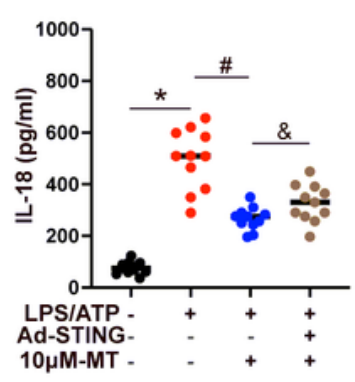

Ad-STING

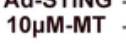

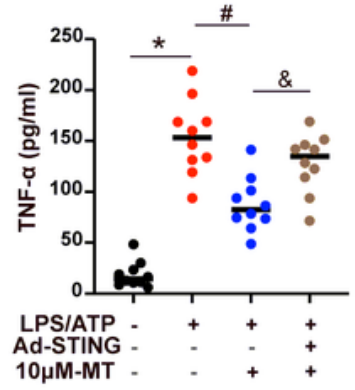
$10 \mu \mathrm{M}-\mathrm{MT}$
D
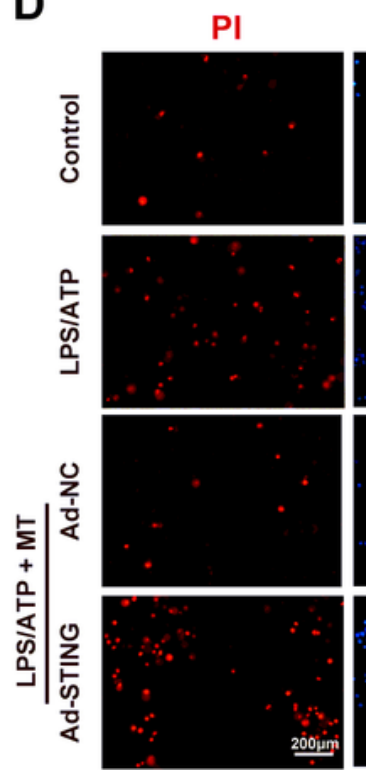

Hoechst
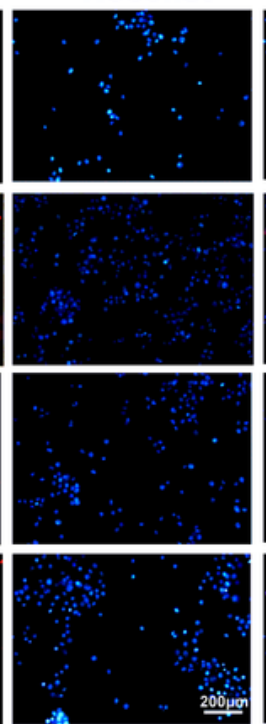

Merge
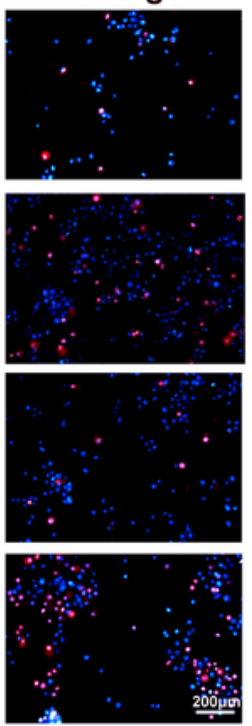

E

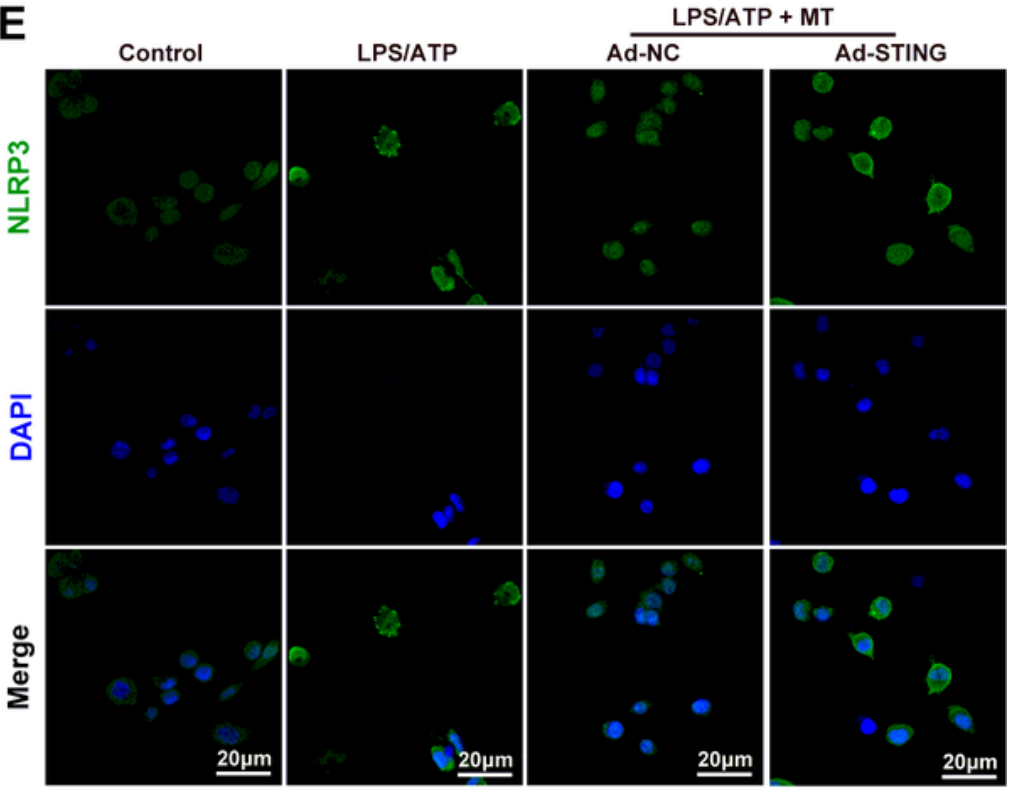

Figure 6 
Over-expression of STING reversed the anti-pyroptotic effect of melatonin $(10 \mu \mathrm{M})$ on LPS/ATP induced BV2 cells. A. The Representative Western blotting of pyroptosis-associated proteins (NLRP3, GSDMD, GSDMD-NT, Cleaved Caspase-1) and quantitative analysis in different groups (Control, LPS/ATP, LPS/ATP+10 $\mu M$ MT and LPS/ATP+10 $\mu M M T+A d-S T I N G) ; B$. The relative released LDH level in the cell culture supernatant of different groups assessed by LDH kits; $\mathrm{C}$. The level of pro-inflammatory cytokine (TNF-a, IL-1 $\beta$ and IL-18) in cell culture supernatant of different groups detected by commercial ELISA kits; D. The representative images of PI staining of BV2 cells in different groups, Nuclei are stained with Hoechst (blue), scale bar: $200 \mu \mathrm{m}$; $\mathrm{E}$. The representative immunofluorescence images of NLRP3 (green) in BV2 cells of different groups. Nuclei are stained with DAPI (blue), Scale bar: $20 \mu \mathrm{m} .{ }^{*} p<0.05,{ }^{*} p<0.01$ versus Control group, $\# p<0.05$ versus LPS/ATP group, \&p $<0.05$ versus LPS/ATP+10 $4 M$ MT group. All data were shown as the mean \pm SD from 3 independent experiments. 
A
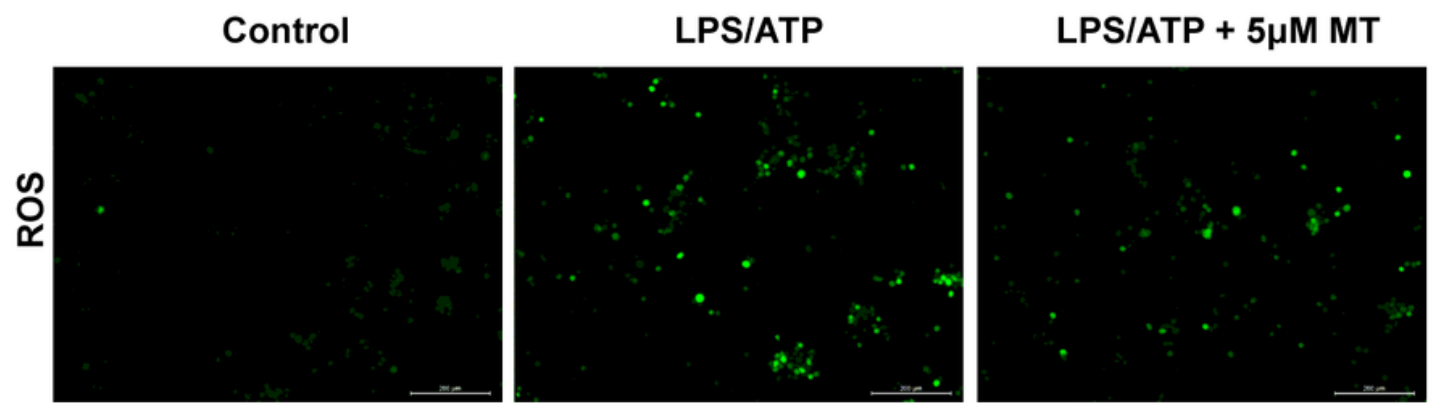

LPSIATP + 10 $\mu M M T$
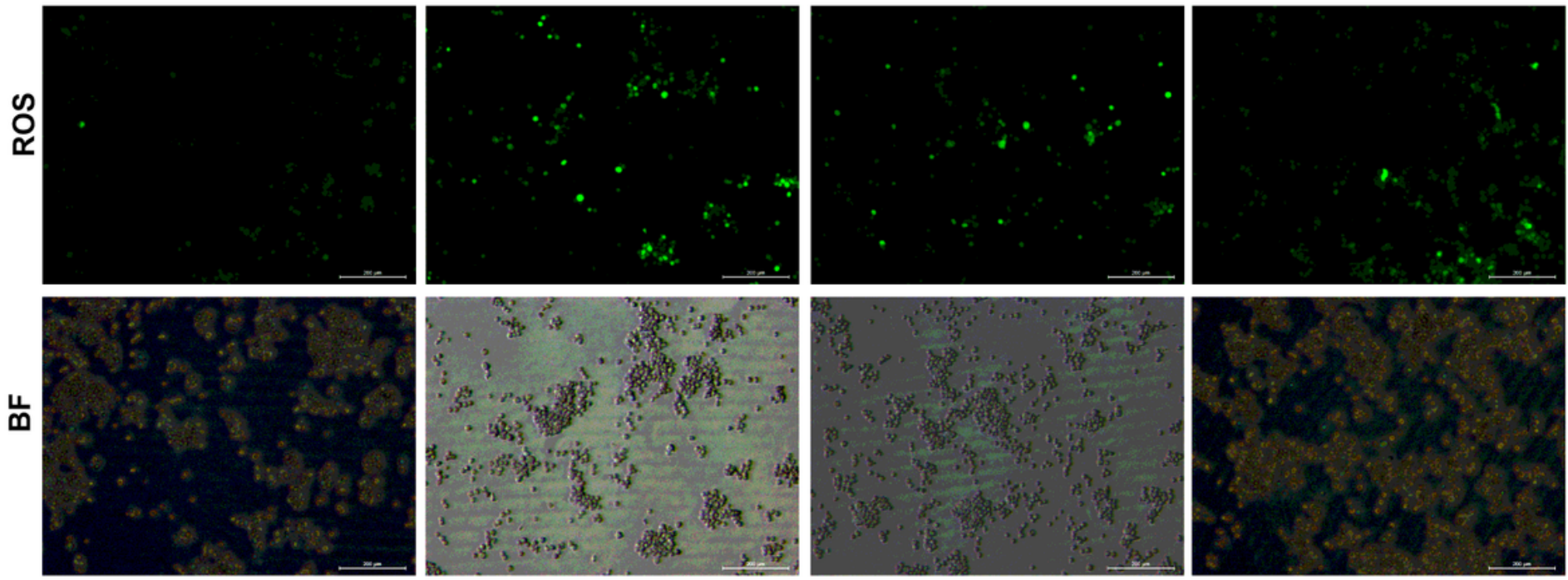

B

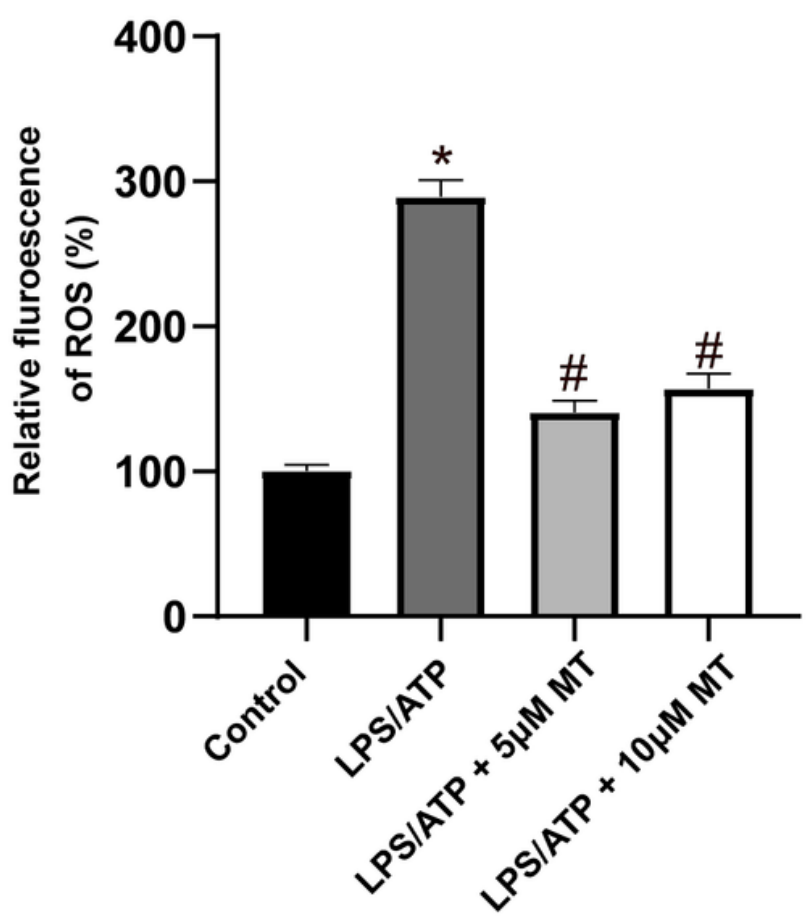

C

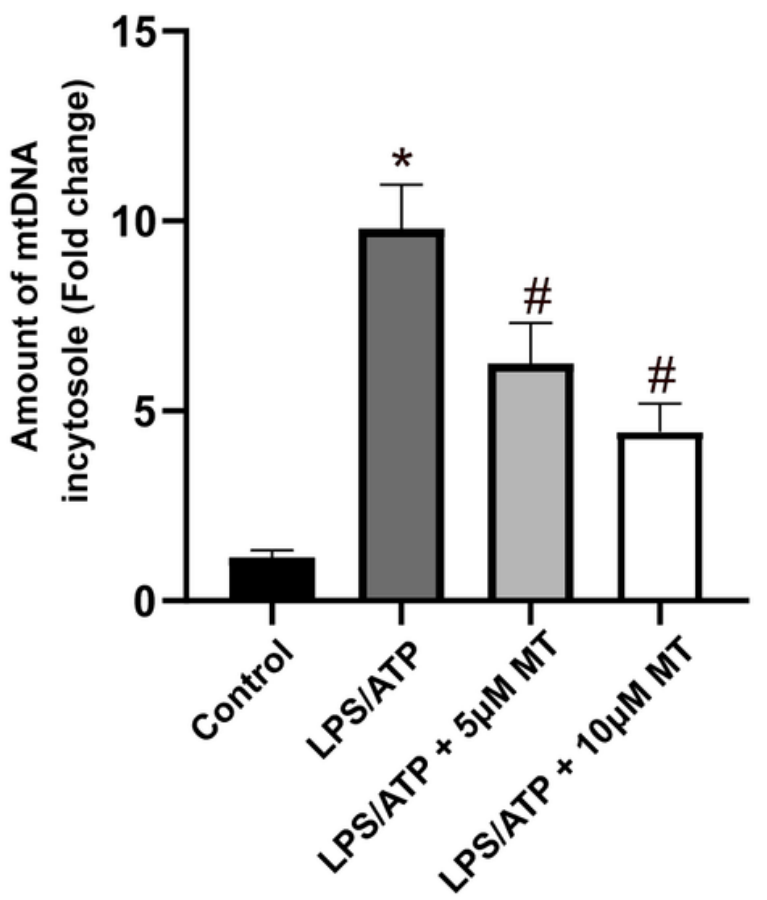

Figure 7

Melatonin reduced the ROS production and the release of mtDNA in LPS/ATP-induced BV2 cells. A. The representative immunofluorescence images of ROS detected by DCFH-DA fluorescence probe in BV2 cells of four groups (Control, LPS/ATP, LPS/ATP $+5 \mu M$ MT and LPS/ATP+10 $\mu M$ MT). Scale bar: $200 \mu m$. B. The quantitative results of ROS production in different four groups. $C$. The relative level of cytosolic mtDNA in BV2 cells of different four groups. ${ }^{*} p<0.05$ versus Control group, $\# p<0.05$ versus LPS/ATP group. All data were shown as the mean \pm SD from 3 independent experiments. 

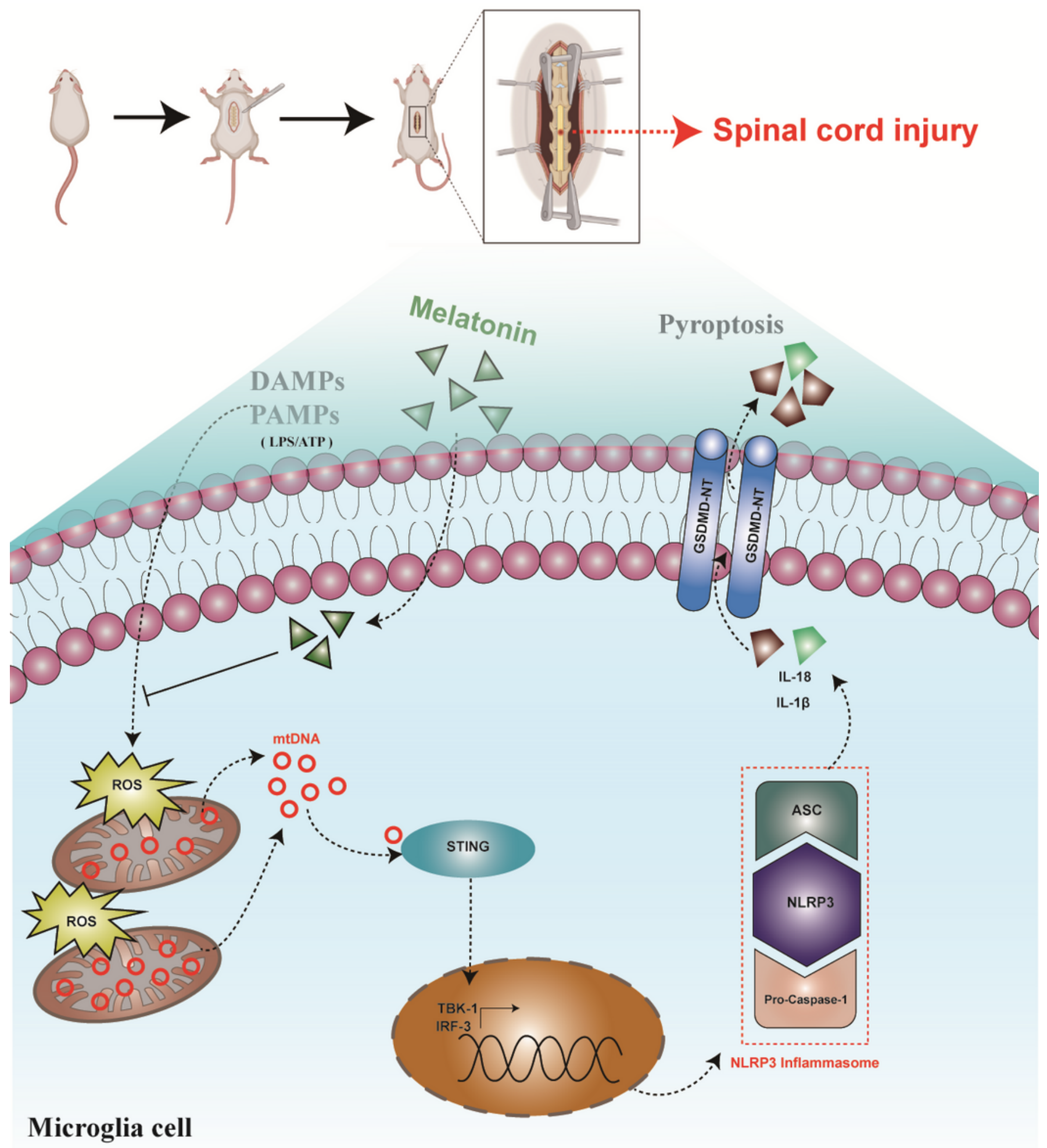

\section{Figure 8}

The mechanism of anti-pyroptotic effect of melatonin on spinal cord injury. Melatonin reduced the DAMPs/PAMPs induced ROS production and the mtDNA release in microglia cells, subsequently blocked the activation of STING pathway. Thereafter, the NLRP3 inflammasome-mediated pyroptosis were inhibited and the corresponding neuroinflammation were alleviated during the secondary phase of SCl. 